\title{
Paraproduit sur le groupe de Heisenberg et applications
}

\author{
Hajer Bahouri et Isabelle Gallagher
}

Résumé. En adaptant au cas inhomogène la décomposition de Littlewood-Paley homogène sur le groupe de Heisenberg introduite par H. Bahouri, P. Gérard et C.-J. Xu dans [4], on construit des opérateurs de paraproduit analogues à ceux définis par J.-M. Bony dans [5]; malgré le fait que l'on ne dispose pas de formule simple pour la transformée de Fourier d'un produit, des propriétés de localisation spectrale du cas classique sont préservées sur le groupe de Heisenberg après passage au produit. À partir du découpage dyadique et du paraproduit, on démontre l'inégalité de Gagliardo-Nirenberg sur le groupe de Heisenberg, et l'on étudie la régularité des solutions de systèmes sous-elliptiques semilinéaires, ainsi que des équations d'ondes semi-linéaires.

Abstract. We adapt the homogeneous Littlewood-Paley decomposition on the Heisenberg group constructed by H. Bahouri, P. Gérard and C.-J. $\mathrm{Xu}$ in [4] to the inhomogeneous case, which enables us to build paraproduct operators, similar to those defined by J.-M. Bony in [5]; although there is no simple formula for the Fourier transform of the product of two functions, some spectral localization properties of the classical case are preserved on the Heisenberg group after the product has been taken. Using the dyadic decomposition and the paraproduct algorithm, we prove the Gagliardo-Nirenberg inequality on the Heisenberg group; the smoothness of solutions of subelliptic, semi-linear systems is also studied, as well as semi-linear wave equations. 


\section{Introduction.}

Ce travail vise à donner des applications de la théorie de Littlewood-Paley sur le groupe de Heisenberg, à partir de la construction de la décomposition homogène menée par H. Bahouri, P. Gérard et C.- J. $\mathrm{Xu}$ dans [4]. Une adaptation de la méthode de [4], utilisant aussi un résultat de [11], conduit à la construction d'une décomposition inhomogène, ce qui nous permet de transposer au groupe de Heisenberg divers résultats connus dans le cas classique (que l'on peut trouver par exemple dans [5], [6] ou dans [7]), concernant la théorie de LittlewoodPaley. On démontre notamment des résultats concernant le coût de la dérivation pour des fonctions dont la transformée de Fourier est localisée dans une boule ou dans une couronne, ainsi que des estimations concernant l'action des applications homogènes et la composition par des fonctions $C^{\infty}$. Enfin cette décomposition de Littlewood-Paley permet de démontrer les inclusions de Sobolev $H^{s} \subset L^{p}-$ ces inclusions sont également démontrées dans [4], par une méthode différente.

On définit ensuite, dans la Section 4, les opérateurs de paraproduit sur le groupe de Heisenberg, et l'on étudie leurs propriétés. La définition de J.-M. Bony (voir [5]) dans le cas classique s'avère opérante dans ce cadre, une fois vérifié (par la Proposition 4.1) que certaines propriétés de localisation dans l'espace de Fourier sont préservées après passage au produit. Ces opérateurs, comme dans le cas classique, permettent de démontrer des lois de produit et des estimations douces dans les espaces de Besov.

Enfin la dernière section est consacrée à des applications de cette théorie. La première concerne la démonstration de l'inégalité de Gagliardo-Nirenberg par utilisation du découpage dyadique et des fonctions maximales sur le groupe de Heisenberg. La seconde application vise à démontrer un résultat de régularité pour les solutions d'équations sous-elliptiques semi-linéaires, par utilisation du paraproduit. La méthode de démonstration suit la démarche de J.-Y. Chemin et C.-J. Xu dans [8]. Enfin la dernière application concerne des équations d'ondes semi-linéaires sur le groupe de Heisenberg: en utilisant les estimations douces démontrées plus haut, associées aux estimations de Strichartz généralisées de [4], on démontre un théorème analogue à un résultat de G. Ponce et T. Sideris (voir [17]) dans le cas classique.

Remarquons que dans [12], P.-G. Lemarié construit une base d'ondelettes sur les groupes de Lie nilpotents stratifiés abstraits, à partir de laquelle on peut déduire une formule de paraproduit. L'intérêt de 
la construction que nous présentons ici est qu'elle est adaptée au cadre Heisenberg, et ainsi directement utilisable pour des applications.

\section{Notations et rappels.}

Nous allons rappeller ici quelques résultats sur la théorie de Littlewood-Paley sur le groupe de Heisenberg. Pour des détails concernant le groupe de Heisenberg, nous renvoyons à [9], [14], [15], [16], [18], [19], et pour le découpage dyadique homogène sur le groupe de Heisenberg, on consultera le travail de H. Bahouri, P. Gérard et C.-J. Xu dans [3] et $[4]$.

\subsection{Rappels de définitions.}

Le groupe de Heisenberg $\mathbb{H}^{n}$ est l'ensemble $\mathbb{C}^{n} \times \mathbb{R}$ muni de la loi de produit suivante

$$
(z, s) \cdot\left(z^{\prime}, s^{\prime}\right)=\left(z+z^{\prime}, s+s^{\prime}+2 \operatorname{Im} z \cdot \bar{z}^{\prime}\right),
$$

pour tous $\left((z, s),\left(z^{\prime}, s^{\prime}\right)\right) \in \mathbb{H}^{n} \times \mathbb{H}^{n}$. Le groupe $\mathbb{H}^{n}$ étant non commutatif, la transformée de Fourier sur $\mathbb{H}^{n}$ est définie à l'aide des représentations irréductibles unitaires de $\mathbb{H}^{n}$. Nous choisissons ici les représentations définies à partir des espaces de Bargmann,

$$
\mathcal{H}_{\lambda}=\left\{F \text { holomorphe sur } \mathbb{C}^{n},\|F\|_{\mathcal{H}_{\lambda}}<\infty\right\},
$$

où l'on a noté

$$
\|F\|_{\mathcal{H}_{\lambda}}^{2} \stackrel{\text { def }}{=}\left(\frac{2|\lambda|}{\pi}\right)^{n} \int_{\mathbb{C}^{n}} e^{-2|\lambda||\xi|^{2}}|F(\xi)|^{2} d \xi,
$$

et les représentations irréductibles unitaires $\left(u^{\lambda}, \mathcal{H}_{\lambda}\right)_{\lambda \neq 0}$ sont alors

$$
u_{z, s}^{\lambda} F(\xi)=F(\xi-\bar{z}) e^{i \lambda s+2 \lambda\left(\xi \cdot z-|z|^{2} / 2\right)}, \quad \text { pour } \lambda>0,
$$

et

$$
u_{z, s}^{\lambda} F(\xi)=F(\xi+z) e^{i \lambda s+2 \lambda\left(\xi \cdot \bar{z}-|z|^{2} / 2\right)}, \quad \text { pour } \lambda<0 .
$$

Notons que l'on a une base orthornormée de l'espace de Hilbert $\mathcal{H}_{\lambda}$, formée de

$$
F_{\alpha, \lambda}(\xi)=\frac{(\sqrt{2|\lambda|} \xi)^{\alpha}}{\sqrt{\alpha}}, \quad \alpha \in \mathbb{N}
$$


On définit la transformée de Fourier d'une fonction $f \in L^{1}\left(\mathbb{H}^{n}\right)$ par

$$
\mathcal{F}(f)(\lambda)=\int_{\mathbb{H}^{n}} f(z, s) u_{z, s}^{\lambda} d z d s
$$

Dans le cas particulier des fonctions radiales, telles que $f(z, s)=g(|z|, s)$, la proposition suivante, démontrée dans [15], nous sera d'une grande utilité.

Proposition 2.1. Si $f \in L^{2}\left(\mathbb{H}^{n}\right)$ est radiale, alors $\mathcal{F}(f)(\lambda) F_{\alpha, \lambda}=$ $R_{|\alpha|}(\lambda) F_{\alpha, \lambda}$, où

$R_{m}(\lambda)=\left(\begin{array}{c}m+n-1 \\ m\end{array}\right)^{-1} \int f(z, s) e^{i \lambda s} L_{m}^{(n-1)}\left(2|\lambda||z|^{2}\right) e^{-|\lambda||z|^{2}} d z d s$ et où les $L_{m}^{(n)}(t)$ sont les polynômes de Laguerre

$$
L_{m}^{(n)}(t)=\sum_{k=0}^{m}(-1)^{k}\left(\begin{array}{c}
m+n \\
m-k
\end{array}\right) \frac{t^{k}}{k !}
$$

Réciproquement, s'il existe des scalaires $R_{m}(\lambda)$ tels que $\mathcal{F}(f)(\lambda) F_{\alpha, \lambda}=$ $R_{|\alpha|}(\lambda) F_{\alpha, \lambda}$, et

$$
\sum_{m}\left(\begin{array}{c}
m+n-1 \\
m
\end{array}\right)^{-1} \int\left|R_{m}(\lambda)\right|^{2}|\lambda|^{n} d \lambda<\infty
$$

alors $f \in L^{2}\left(\mathbb{H}^{n}\right)$ est radiale, et l'on a presque partout

$$
f(z, s)=\frac{2^{n-1}}{\pi^{n+1}} \sum_{m} \int e^{-i \lambda s} R_{m}(\lambda) L_{m}^{(n-1)}\left(2|\lambda||z|^{2}\right) e^{-|\lambda||z|^{2}}|\lambda|^{n} d \lambda .
$$

Enfin rappellons qu'il existe une base de champs de vecteurs invariants à gauche sur le groupe de Heisenberg, notés

$X_{j}=\partial_{x_{j}}+2 y_{j} \partial_{s} \quad$ et $\quad Y_{j}=\partial_{y_{j}}-2 x_{j} \partial_{s}, \quad$ pour tous $j \in\{1, \ldots, n\}$, où l'on a écrit, pour tout $z_{j} \in \mathbb{C}^{n}, z_{j}=x_{j}+i y_{j}$. On notera

$$
\Delta_{\mathbb{H}^{n}} \stackrel{\text { def }}{=} \sum_{j=1}^{n}\left(X_{j}^{2}+Y_{j}^{2}\right)
$$


et pour tout $\gamma \in \mathbb{N}, \mathcal{X}^{\gamma}$ sera un produit de $\gamma$ champs de vecteurs, du type

$$
\mathcal{X}^{\gamma}=X_{j_{1}} \cdots X_{j_{\ell}}
$$

où $j_{k} \in\{1, \ldots, 2 n\}$, et l'on convient que $X_{j+n}=Y_{j}$, pour $j \in\{1, \ldots, n\}$. Remarquons que pour toute fonction $f \in \mathcal{S}\left(\mathbb{H}^{n}\right)$

$$
\mathcal{F}\left(\Delta_{\mathbb{H} n} f\right)(\lambda) F_{\alpha, \lambda}=-4|\lambda|(2|\alpha|+n) \mathcal{F}(f)(\lambda) F_{\alpha, \lambda} .
$$

On peut enfin définir l'opérateur suivant

$$
\mathcal{F}\left(\left(-\Delta_{\mathbb{H}_{n}}\right)^{\rho / 2} f\right)(\lambda) F_{\alpha, \lambda}=(4|\lambda|(2|\alpha|+n))^{\rho / 2} \mathcal{F}(f)(\lambda) F_{\alpha, \lambda},
$$

pour tous $\rho \in \mathbb{R}, f \in \mathcal{S}\left(\mathbb{H}^{n}\right)$.

\subsection{Théorie de Littlewood-Paley sur le groupe de Heisenberg.}

Nous allons rappeller tout d'abord la définition de la décomposition de Littlewood-Paley homogène construite dans [3] et [4]. Nous préciserons à la fin de ce paragraphe comment cette construction peut s'adapter pour obtenir une décomposition inhomogène.

Dorénavant nous noterons $\mathcal{C}_{0}$ la couronne $\{\tau \in \mathbb{R}: 1 / 2 \leq|\tau| \leq 4\}$, $\mathcal{B}_{0}$ la boule $\{\tau \in \mathbb{R}:|\tau| \leq 2\}$, et nous considèrerons une fonction $R^{*} \in C_{0}^{\infty}\left(\mathcal{C}_{0}\right)$, telle que

$$
\sum_{j \in \mathbb{Z}} R^{*}\left(2^{-2 j} \tau\right)=1, \quad \text { pour tout } \tau \in \mathbb{R}^{*} .
$$

D'autre part, on définit la fonction $\widetilde{R}^{*} \in C_{0}^{\infty}\left(\mathcal{B}_{0}\right)$, identiquement égale à 1 près de 0 , telle que

$$
\widetilde{R}^{*}(\tau)+\sum_{j \geq 0} R^{*}\left(2^{-2 j} \tau\right)=1, \quad \text { pour tout } \tau \in \mathbb{R} .
$$

Dans ce qui suit, nous noterons $R_{m}^{*}(\tau)=R^{*}((2 m+n) \tau)$. La Proposition 2.1 nous permet de définir la fonction radiale

$$
\varphi(z, s)=\frac{2^{n-1}}{\pi^{n+1}} \sum_{m} \int e^{-i \lambda s} R_{m}^{*}(\lambda) L_{m}^{(n-1)}\left(2|\lambda||z|^{2}\right) e^{-|\lambda||z|^{2}}|\lambda|^{n} d \lambda,
$$


et [4, Proposition 2.2] indique que $\varphi$ est un élément de $\mathcal{S}\left(\mathbb{H}^{n}\right)$. Ce résultat est aussi une conséquence de [11]. Ainsi l'on peut écrire en particulier

$$
\mathcal{F}(\varphi)(\lambda) F_{\alpha, \lambda}=R_{|\alpha|}^{*}(\lambda) F_{\alpha, \lambda},
$$

et si $\varphi_{j}(z, s)=2^{N j} \varphi\left(2^{j} z, 2^{2 j} s\right)$, où $N=2 n+2$ est la dimension homogène de $\mathbb{H}^{n}$, alors la série

$$
f=\sum_{j \in \mathbb{Z}} \dot{\Delta}_{j} f, \quad \text { avec } \quad \dot{\Delta}_{j} f=f * \varphi_{j},
$$

est la décomposition de Littlewood-Paley de $f \in \mathcal{S}\left(\mathbb{H}^{n}\right)$ sur le groupe de Heisenberg.

La convergence de la série $\sum_{j \in \mathbb{Z}} \dot{\Delta}_{j} f$ est démontrée dans [4]. Notons que cette série ne converge pas dans $\mathcal{S}^{\prime}\left(\mathbb{H}^{n}\right)$ (à cause des fonctions polynômiales, qui vérifient $\dot{\Delta}_{j} f=0$, pour tout $j \in \mathbb{Z}$ ). D'autre part, notons que cette décomposition dyadique est bien une décomposition de Littlewood-Paley, puisque (voir [4, Proposition 2.3]) si $f \in \mathcal{S}^{\prime}\left(\mathbb{H}^{n}\right)$ vérifie $\sum_{j \in \mathbb{Z}} \dot{\Delta}_{j} f=f$, alors $f \in L^{p}\left(\mathbb{H}^{n}\right)$ est équivalent à $\left\|\dot{\Delta}_{j} f\right\|_{\ell^{2}(\mathbb{Z})} \in$ $L^{p}\left(\mathbb{H}^{n}\right)$.

Notre but étant d'écrire une théorie du paraproduit sur le groupe de Heisenberg, il convient à présent de construire une décomposition inhomogène de Littlewood-Paley; en d'autres termes, nous allons à présent chercher à montrer que la fonction $\psi$ définie par

$$
\begin{aligned}
& \psi(z, s) \\
& \qquad=\frac{2^{n-1}}{\pi^{n+1}} \sum_{m} \int e^{-i \lambda s} \widetilde{R}_{m}^{*}(\lambda) L_{m}^{(n-1)}\left(2|\lambda||z|^{2}\right) e^{-|\lambda||z|^{2}}|\lambda|^{n} d \lambda
\end{aligned}
$$

vérifie $\psi \in \mathcal{S}\left(\mathbb{H}^{n}\right)$. Alors on écrira, pour $f \in L^{2}\left(\mathbb{H}^{n}\right)$,

$$
\begin{aligned}
& f=\sum_{j \geq-1} \Delta_{j} f, \quad \text { avec pour tout } j \in \mathbb{N}, \\
& \Delta_{j} f=\dot{\Delta}_{j} f, \quad \Delta_{-1} f=f * \psi \\
& \text { et pour tout } j<-1, \quad \Delta_{j} f=0 .
\end{aligned}
$$

On définit aussi $S_{j} f=\psi_{j} * f$, avec $\psi_{j}(z, s)=2^{N j} \psi\left(2^{j} z, 2^{2 j} s\right)$, pour tout $j \in \mathbb{N}$.

Il s'agit donc de vérifier que $\psi$ définie en (2.4) est un élément de $\mathcal{S}\left(\mathbb{H}^{n}\right)$. Une adaptation de [4, Proposition 2.2] nous permet de démontrer le résultat suivant. 
Proposition 2.2. La fonction $\psi$ définie par

$$
\psi(z, s)=\frac{2^{n-1}}{\pi^{n+1}} \sum_{m} \int e^{-i \lambda s} \widetilde{R}_{m}^{*}(\lambda) L_{m}^{(n-1)}\left(2|\lambda||z|^{2}\right) e^{-|\lambda||z|^{2}}|\lambda|^{n} d \lambda,
$$

où $\widetilde{R}^{*} \in C_{0}^{\infty}\left(\mathcal{B}_{0}\right)$ est identiquement égale à 1 près de 0 , vérifie

$$
\begin{gathered}
\left\|\left(-\Delta_{\mathbb{H}^{n}}\right)^{k} \psi\right\|_{L^{2}\left(\mathbb{H}^{n}\right)} \leq C_{k}, \quad \text { pour tout } k \in \mathbb{N}, \\
\left\|\left(i s-|z|^{2}\right)^{\ell} \psi\right\|_{L^{2}\left(\mathbb{H}^{n}\right)} \leq C_{\ell}, \quad \text { pour tout } \ell \in \mathbb{N},
\end{gathered}
$$

et donc $\psi$ est dans $\mathcal{S}\left(\mathbb{H}^{n}\right)$.

DÉmonstration de la Proposition. Nous allons commencer par démontrer (2.6). Rappellons qu'il est démontré dans [4] que la transformée de Fourier sur le groupe de Heisenberg réalise un isomorphisme du sous-espace des fonctions radiales de $L^{2}\left(\mathbb{H}^{n}\right)$ sur les opérateurs $A$ à un paramètre, définis par

$$
A(\lambda) F_{\alpha, \lambda}=Q_{|\alpha|}(\lambda) F_{\alpha, \lambda}
$$

avec

$$
\frac{2^{n-1}}{\pi^{n+1}} \sum_{m}\left(\begin{array}{c}
m+n-1 \\
m
\end{array}\right) \int_{-\infty}^{\infty}\left|Q_{m}(\lambda)\right|^{2}|\lambda|^{n} d \lambda<\infty .
$$

Alors la Proposition 2.1, associée à (2.3), donne le résultat, puisque

$$
\begin{gathered}
\sum_{m}\left(\begin{array}{c}
m+n-1 \\
m
\end{array}\right) \int_{-\infty}^{\infty}|-4((2 m+n)|\lambda|)|^{k}\left|\widetilde{R}^{*}((2 m+n) \lambda)\right|^{2}|\lambda|^{n} d \lambda \\
=\sum_{m}\left(\begin{array}{c}
m+n-1 \\
m
\end{array}\right)(2 m+n)^{-n-1} \int_{-\infty}^{\infty}\left|\widetilde{R}^{*, k}(\lambda)\right|^{2}|\lambda|^{n} d \lambda
\end{gathered}
$$

où $\widetilde{R}^{*, k}$ est une fonction de $C_{0}^{0}(\mathbb{R})$, donc cette série est convergente.

Pour ce qui est de (2.7), on peut reprendre les calculs de [4]: si $Q$ est une fonction de $C_{0}^{\infty}\left(\mathbb{R}^{*}\right)$, et si $Q_{m}(\lambda)=Q((2 m+n) \lambda)$, alors il est montré dans [4] que la fonction

$$
f(z, s)=\frac{2^{n-1}}{\pi^{n+1}} \sum_{m} \int e^{-i \lambda s} Q_{m}(\lambda) L_{m}^{(n-1)}\left(2|\lambda||z|^{2}\right) e^{-|\lambda||z|^{2}}|\lambda|^{n} d \lambda
$$


est dans $L^{2}\left(\mathbb{H}^{n}\right)$, mais aussi toutes les fonctions $\left(i s-|z|^{2}\right)^{\ell} f$ pour $\ell \in \mathbb{N}$. On constate facilement (nous n'entrerons pas dans les détails ici), au vu de la démonstration de ce résultat dans [4], que la condition $Q \in C_{0}^{\infty}\left(\mathbb{R}^{*}\right)$ peut être relaxée en $Q \in C_{0}^{\infty}(\mathbb{R})$, et $Q$ constante près de $\lambda=0$. C'est en particulier le cas pour la fonction $\widetilde{R}^{*}$, et donc le point (2.7) est démontré.

Reste donc à vérifier que (2.6) et (2.7) impliquent bien que $\psi \in$ $\mathcal{S}\left(\mathbb{H}^{n}\right)$. Cela résulte du lemme suivant.

Lemme 2.1. Soit $f \in \mathcal{S}^{\prime}\left(\mathbb{H}^{n}\right)$ telle que

$$
\left(-\Delta_{\mathbb{H}^{n}}\right)^{k} f \in L^{2}\left(\mathbb{H}^{n}\right), \quad \text { pour tout } k \in \mathbb{N},
$$

et

$$
\left(i s-|z|^{2}\right)^{\ell} f \in L^{2}\left(\mathbb{H}^{n}\right), \quad \text { pour tout } \ell \in \mathbb{N} .
$$

Alors $f \in \mathcal{S}\left(\mathbb{H}^{n}\right)$.

DÉmonstration. Nous allons démontrer ce résultat uniquement dans le cas $\ell=1$; le cas général s'en déduit sans difficulté. La sous-ellipticité de $\left(-\Delta_{\mathbb{H}^{n}}\right)^{k}$ implique (voir [4, Lemme 2.1]) qu'il suffit de démontrer que

$$
\left(-\Delta_{\mathbb{H} n}\right)^{k}\left(\left(i s-|z|^{2}\right) f\right) \in L^{2}\left(\mathbb{H}^{n}\right), \quad \text { pour tout } k \in \mathbb{N} .
$$

Il est facile de voir, par la formule de Leibnitz et avec la notation (2.1), que

$$
\mathcal{X}^{2 k}\left(\left(i s-|z|^{2}\right) f\right)=\left(i s-|z|^{2}\right) \mathcal{X}^{2 k} f+\sum_{k^{\prime}<2 k} P_{k^{\prime}}(z) \mathcal{X}^{k^{\prime}} f
$$

pour tout $k \in \mathbb{N}^{*}$, où $P_{k^{\prime}}$ est un polynôme. Mais l'hypothèse ( $i s$ $\left.|z|^{2}\right)^{\ell} f \in L^{2}\left(\mathbb{H}^{n}\right)$ implique que pour tout $\beta \in \mathbb{N}$, on a $z^{\beta} f \in L^{2}\left(\mathbb{H}^{n}\right)$, par conséquent il vient

$$
\begin{aligned}
\int_{\mathbb{H}^{n}} \mathcal{X}^{2 k}\left(\left(i s-|z|^{2}\right) f\right) \mathcal{X}^{2 k}\left(\left(i s-|z|^{2}\right) f\right) d z d s \\
=C \int_{\mathbb{H}^{n}}\left(i s-|z|^{2}\right) f \mathcal{X}^{4 k}\left(\left(i s-|z|^{2}\right) f\right) d z d s \\
=C \int_{\mathbb{H}^{n}}\left(i s-|z|^{2}\right)^{2} f \mathcal{X}^{4 k} f d z d s
\end{aligned}
$$




$$
\begin{aligned}
& +\sum_{k^{\prime}<4 k} \int_{\mathbb{H}^{n}} P_{k^{\prime}}(z)\left(i s-|z|^{2}\right) f \mathcal{X}^{k^{\prime}} f d z d s \\
\leq & C \sup _{\beta \leq 2 k}\left\|\left(-\Delta_{\mathbb{H}^{n}}\right)^{\beta} f\right\|_{L^{2}\left(\mathbb{H}^{n}\right)}\left\|\left(i s-|z|^{2}\right)^{\beta} f\right\|_{L^{2}\left(\mathbb{H}^{n}\right)} \cdot
\end{aligned}
$$

Le lemme est démontré, et avec lui, la proposition.

\section{Lemme de localisation et applications.}

\section{1. Énoncé du lemme et démonstration.}

Le résultat suivant est l'analogue du [6, Lemme 2.1.1] dans le cas classique. Il décrit le coût de la dérivation pour une fonction dont la transformée de Fourier est localisée.

Lemme 3.1. Soient $p$ et $q$ deux éléments de $[1, \infty]$, avec $p \leq q$, et soit $u \in L^{p}\left(\mathbb{H}^{n}\right)$ une fonction telle que $u * f=0$ pour toute fonction radiale $f \in \mathcal{S}\left(\mathbb{H}^{n}\right)$ vérifiant, pour tout $\alpha \in \mathbb{N}^{n}$,

$$
\mathcal{F}(f)(\lambda) F_{\alpha, \lambda}=0, \quad \text { pour } \lambda \in(2|\alpha|+n)^{-1} 2^{2 j} \mathcal{B}_{0} .
$$

Alors on a

$$
\sup _{\beta=k}\left\|\mathcal{X}^{\beta} u\right\|_{L^{q}\left(\mathbb{H}^{n}\right)} \leq C_{k} 2^{N j(1 / p-1 / q)+k j}\|u\|_{L^{p}\left(\mathbb{H}^{n}\right)},
$$

pour tout $k \in \mathbb{N}$. D'autre part, si $u * g=0$ pour toute fonction radiale $g \in \mathcal{S}\left(\mathbb{H}^{n}\right)$ vérifiant, pour tout $\alpha \in \mathbb{N}^{n}$,

$$
\mathcal{F}(g)(\lambda) F_{\alpha, \lambda}=0, \quad \text { pour } \lambda \in(2|\alpha|+n)^{-1} 2^{2 j} \mathcal{C}_{0},
$$

alors

$$
C_{\rho}^{-1} 2^{-j \rho}\left\|\left(-\Delta_{\mathbb{H}^{n}}\right)^{\rho / 2} u\right\|_{L^{p}\left(\mathbb{H}^{n}\right)} \leq\|u\|_{L^{p}\left(\mathbb{H}^{n}\right)}
$$

$$
\leq C_{\rho} 2^{-j \rho}\left\|\left(-\Delta_{\mathbb{H}^{n}}\right)^{\rho / 2} u\right\|_{L^{p}\left(\mathbb{H}^{n}\right)},
$$

pour tout $\rho \in \mathbb{R}$.

Remarques. Dans le cas où la fonction $u$ est un élément de $\mathcal{S}\left(\mathbb{H}^{n}\right)$, alors les hypothèses (3.8) et (3.10) se traduisent respectivement en

$$
\mathcal{F}(u)(\lambda) F_{\alpha, \lambda}=\mathbf{1}_{(2|\alpha|+n)^{-1} 2^{2 j} \mathcal{B}_{0}}(\lambda) \mathcal{F}(u)(\lambda) F_{\alpha, \lambda},
$$


et

$$
\mathcal{F}(u)(\lambda) F_{\alpha, \lambda}=\mathbf{1}_{(2|\alpha|+n)^{-1} 2^{2 j} \mathcal{C}_{0}}(\lambda) \mathcal{F}(u)(\lambda) F_{\alpha, \lambda} .
$$

Notons en outre que le second résultat de ce lemme ne concerne que le cas où l'opérateur de dérivation est du type $\left(-\Delta_{\mathbb{H}^{n}}\right)^{\rho / 2}$. Cela est dû au fait que dans le cas des opérateurs $X_{j}$, on ne dispose pas de décomposition dans la base des $F_{\alpha, \lambda}$ aussi simple que celle donnée par (2.2) pour $-\Delta_{\mathbb{H}^{n}}$.

DÉmonstration Du Lemme. Nous allons nous placer dans le cas où la fonction $u$ est un élément de $\mathcal{S}\left(\mathbb{H}^{n}\right)$; le lemme suit par densité. Soit $R \in C_{0}^{\infty}(\mathbb{R})$, identiquement égale à 1 près de $\mathcal{B}_{0}$. Alors on a

$$
\mathcal{F}(u)(\lambda) F_{\alpha, \lambda}=R_{|\alpha|}\left(2^{-2 j} \lambda\right) \mathcal{F}(u)(\lambda) F_{\alpha, \lambda},
$$

où l'on a posé $R_{|\alpha|}(\lambda)=R((2|\alpha|+n) \lambda)$. Mais alors d'après les propositions 2.1 et 2.2 , il existe une fonction $g \in \mathcal{S}\left(\mathbb{H}^{n}\right)$ radiale, telle que

$$
\mathcal{F}(g)(\lambda) F_{\alpha, \lambda}=R_{|\alpha|}(\lambda) F_{\alpha, \lambda}
$$

En écrivant $g_{j}(z, s)=2^{N j} g\left(2^{j} z, 2^{2 j} s\right)$, on a alors

$$
\mathcal{F}(u)(\lambda) F_{\alpha, \lambda}=\mathcal{F}\left(g_{j}\right)(\lambda) \mathcal{F}(u)(\lambda) F_{\alpha, \lambda}
$$

et donc $u=g_{j} * u$. Mais on a alors

$$
\mathcal{X}^{\beta} u=2^{j(N+\beta)} \mathcal{X}^{\beta} g\left(\delta_{2^{j}} \cdot\right) * u
$$

où pour tout $a, \delta_{a}$ est la dilatation homogène définie par $\delta_{a}(z, s)=$ $\left(a z, a^{2} s\right)$. Comme dans [6], il suffit alors d'appliquer l'inégalité de Young pour obtenir (3.9).

Démontrons à présent (3.11). Soit $R^{\prime} \in C_{0}^{\infty}\left(\mathbb{R}^{*}\right)$, identiquement égale à 1 près de $\mathcal{C}_{0}$. Alors

$$
\mathcal{F}(u)(\lambda) F_{\alpha, \lambda}=R_{|\alpha|}^{\prime}\left(2^{-2 j} \lambda\right) \mathcal{F}(u)(\lambda) F_{\alpha, \lambda},
$$

où $R_{|\alpha|}^{\prime}(\lambda)=R^{\prime}((2|\alpha|+n) \lambda)$, donc

$$
\mathcal{F}(u)(\lambda) F_{\alpha, \lambda}=2^{-j \rho} \frac{R_{|\alpha|}^{\prime}\left(2^{-2 j} \lambda\right)}{\left(42^{-2 j}|\lambda|(2|\alpha|+n)\right)^{\rho / 2}} \mathcal{F}\left(\left(-\Delta_{\mathbb{H}^{n}}\right)^{\rho / 2} u\right)(\lambda) F_{\alpha, \lambda} .
$$


Définissons alors la fonction

$$
\theta^{\rho}(\lambda)=\frac{R^{\prime}(\lambda)}{2^{\rho}|\lambda|^{\rho / 2}},
$$

à laquelle on associe $\theta_{|\alpha|}^{\rho}(\lambda)=\theta^{\rho}((2|\alpha|+n) \lambda)$. Alors $\theta^{\rho} \in C_{0}^{\infty}\left(\mathbb{R}^{*}\right)$, et d'après [4], il existe une fonction $g^{\rho} \in \mathcal{S}\left(\mathbb{H}^{n}\right)$ telle que

$$
\mathcal{F}\left(g^{\rho}\right)(\lambda) F_{\alpha, \lambda}=\theta_{|\alpha|}^{\rho}(\lambda) F_{\alpha, \lambda},
$$

et l'on conclut comme précédemment. Le lemme est démontré.

\subsection{Applications.}

\subsubsection{Lemme de caractérisation.}

Cette première application du Lemme 3.1 permet de caractériser l'appartenance d'une fonction à un espace de Besov; la définition des espaces de Besov sur le groupe de Heisenberg est identique au cas classique (voir [4]). Rappellons simplement que l'espace $B_{p, r}^{\rho}\left(\mathbb{H}^{n}\right)$, pour $\rho \in \mathbb{R}$ et $(p, r) \in[1, \infty]^{2}$ est défini comme l'espace des distributions tempérées vérifiant

$$
u=\sum_{j} \Delta_{j} u \quad \text { et } \quad\|u\|_{B_{p, r}^{\rho}\left(\mathbb{H}^{n}\right)} \stackrel{\text { def }}{=}\left(\sum_{j \geq-1} 2^{j r \rho}\left\|\Delta_{j} u\right\|_{L^{p}\left(\mathbb{H}^{n}\right)}^{r}\right)^{1 / r}<\infty
$$

et l'espace de Besov homogène $\dot{B}_{p, r}^{\rho}\left(\mathbb{H}^{n}\right)$, pour $\rho<N / p$ est l'espace des distributions tempérées telles que $u=\sum_{j} \dot{\Delta}_{j} u$, et que la norme suivante soit finie

$$
\|u\|_{\dot{B}_{p, r}^{\rho}\left(\mathbb{H}^{n}\right)} \stackrel{\text { def }}{=}\left(\sum_{j \in \mathbb{Z}} 2^{j r \rho}\left\|\dot{\Delta}_{j} u\right\|_{L^{p}\left(\mathbb{H}^{n}\right)}^{r}\right)^{1 / r} .
$$

Remarque. On définit aussi, comme dans le cas classique, les espaces de Hölder $C^{\rho}$, que l'on identifie pour tout $\rho \in \mathbb{R}-\mathbb{N}$ à $B_{\infty, \infty}^{\rho}$, ainsi que les espaces de Sobolev $H^{s}$ et leurs versions homogènes, pour tout $s \in \mathbb{R}$.

Lemme 3.2. Soit $\rho>0$ et $(p, r) \in[1, \infty]^{2}$. Les deux assertions suivantes sont équivalentes. 
i) $u \in B_{p, r}^{\rho}\left(\mathbb{H}^{n}\right)$.

ii) Il existe $\left\{u_{j}\right\}_{j \geq 0}$ telle que $u=\sum u_{j}$, et pour tout $\gamma \in \mathbb{R}$,

$$
\left\|\left(-\Delta_{\mathbb{H}^{n}}\right)^{\gamma} u_{j}\right\|_{L^{p}\left(\mathbb{H}^{n}\right)} \leq C_{\gamma} c_{j} 2^{-j(\rho-2 \gamma)}, \quad \text { avec } \rho>0,
$$

où $C_{\gamma}$ ne dépend que de $\gamma$, et $\left\{c_{j}\right\}_{j \geq 0}$ est une suite de $\ell^{r}(\mathbb{N})$.

DÉmonstration. Le Lemme 3.1 précédent implique clairement que i) implique ii). Démontrons donc que ii) implique i). Soit $j^{\prime} \in \mathbb{N}$, et écrivons

$$
\sum_{j} \Delta_{j^{\prime}} u_{j}=\sum_{j>j^{\prime}} \Delta_{j^{\prime}} u_{j}+\sum_{j \leq j^{\prime}} \Delta_{j^{\prime}} u_{j}
$$

Alors

$$
\begin{aligned}
2^{j^{\prime} \rho} & \left\|\Delta_{j^{\prime}} u\right\|_{L^{p}} \\
& \leq 2^{j^{\prime} \rho} \sum_{j>j^{\prime}}\left\|\Delta_{j^{\prime}} u_{j}\right\|_{L^{p}}+C_{\rho} 2^{j^{\prime} \rho} \sum_{j \leq j^{\prime}} 2^{-2 j^{\prime} \beta}\left\|\Delta_{j^{\prime}}\left(-\Delta_{\mathbb{H} n}\right)^{\beta} u_{j}\right\|_{L^{p}},
\end{aligned}
$$

par le Lemme de localisation 3.1, avec $\beta$ à fixer. Mais alors on a, par hypothèse,

$$
2^{j^{\prime} \rho}\left\|\Delta_{j^{\prime}} u\right\|_{L^{p}} \leq C \sum_{j>j^{\prime}} 2^{\left(j^{\prime}-j\right) \rho} c_{j}+C_{\rho} \sum_{j \leq j^{\prime}} 2^{\left(j-j^{\prime}\right)(2 \beta-\rho)} c_{j},
$$

où $\left\{c_{j}\right\}_{j \geq 0}$ est une suite de $\ell^{r}(\mathbb{N})$. Il suffit alors de prendre la norme $\ell^{r}$ en $j^{\prime}$, en choisissant $\beta$ tel que $2 \beta>\rho$. Comme l'on a en outre supposé que $\rho>0$, on a le résultat.

REMARQUE. Un résultat analogue s'énonce bien sûr dans le cas des espaces homogènes.

Le Lemme 3.1 permet de démontrer de manière évidente la continuité des opérateurs $X_{j}$ dans les espaces de Besov. Les notations sont comme en (2.1).

Lemme 3.3. Soit $\rho<N / p$, et soit le couple $(p, r) \in[1, \infty]$. Si $u$ est un élément de $\dot{B}_{p, r}^{\rho}\left(\mathbb{H}^{n}\right)$, alors pour tout $j \in\{1, \ldots, 2 n\}$, on a $X_{j} u \in \dot{B}_{p, r}^{\rho-1}\left(\mathbb{H}^{n}\right)$, et

$$
\left\|X_{j} u\right\|_{\dot{B}_{p, r}^{\rho-1}\left(\mathbb{H}^{n}\right)} \leq C\|u\|_{\dot{B}_{p, r}^{\rho}\left(\mathbb{H}^{n}\right)} .
$$


DÉmonstration. On a par définition de $\dot{B}_{p, r}^{\rho}\left(\mathbb{H}^{n}\right)$,

$$
\left\|\dot{\Delta}_{k} u\right\|_{L^{p}\left(\mathbb{H}^{n}\right)} \leq c_{k} 2^{-k \rho}, \quad \text { pour tout } k \in \mathbb{Z},
$$

où $\left\{c_{k}\right\}_{k \in \mathbb{Z}}$ est une suite de $\ell^{r}(\mathbb{Z})$. Par conséquent, on a par le lemme d'échantillonage 3.1

$$
\left\|X_{j} \dot{\Delta}_{k} u\right\|_{L^{p}\left(\mathbb{H}^{n}\right)} \leq C c_{k} 2^{-k(\rho-1)}, \quad \text { pour tous } j \in\{1, \ldots, 2 n\}
$$

Les opérateurs $X_{j}$ et $\dot{\Delta}_{k}$ commutent, ce qui démontre le lemme.

REMARQUE. Le même résultat est évidemment vrai dans le cas inhomogène.

\subsubsection{Estimations douces.}

Une autre application du lemme de localisation 3.1 consiste en la démonstration d'estimations douces, du type suivant. Remarquons que les énoncés sont les mêmes dans le cas des espaces homogènes.

Lemme 3.4. Soit $s>0$. Si u et $v$ sont deux éléments de $L^{\infty} \cap H^{s}\left(\mathbb{H}^{n}\right)$, alors $u v$ est un élément de $L^{\infty} \cap H^{s}\left(\mathbb{H}^{n}\right)$, et

$$
\|u v\|_{L^{\infty} \cap H^{s}\left(\mathbb{H}^{n}\right)} \leq C\left(\|u\|_{L^{\infty}}\|v\|_{H^{s}}+\|v\|_{L^{\infty}}\|u\|_{H^{s}}\right) .
$$

Nous ne démontrons pas ce lemme ici, car il peut être obtenu aussi comme un corollaire des lois d'opérance des opérateurs de paraproduit que nous définirons plus bas. Par contre, démontrons le résultat suivant, dont la démonstration dans le cas classique peut être trouvée dans $[1$, p. 100] par exemple.

Lemme 3.5. Soit $k \in \mathbb{N}$, et soient $u$ et $v$ deux fonctions de $L^{\infty}\left(\mathbb{H}^{n}\right) \cap$ $H^{k}\left(\mathbb{H}^{n}\right)$. Alors pour tout couple $(\beta, \gamma) \in \mathbb{N}^{2}$ tel que $\beta+\gamma=k$, on a

$$
\left\|\mathcal{X}^{\beta} u \mathcal{X}^{\gamma} v\right\|_{L^{2}\left(\mathbb{H}^{n}\right)} \leq C\left(\|u\|_{L^{\infty}\left(\mathbb{H}^{n}\right)}\|v\|_{H^{k}\left(\mathbb{H}^{n}\right)}+\|v\|_{L^{\infty}\left(\mathbb{H}^{n}\right)}\|u\|_{H^{k}\left(\mathbb{H}^{n}\right)}\right) .
$$


DÉmonstration. Supposons par exemple que $\beta \geq 1$ (puisque si $\beta=$ $\gamma=0$, alors le résultat est trivial). Par la formule de Leibnitz, on peut écrire

$$
\mathcal{X}^{\beta} u \mathcal{X}^{\gamma} v=\sum_{j} c_{j} X_{j}\left(\mathcal{X}^{\beta_{j}} u \mathcal{X}^{\gamma_{j}} v\right)+c_{k} u \mathcal{X}^{\beta+\gamma} v
$$

où les $c_{j}$ sont des constantes, et où $\beta_{j}+\gamma_{j}=k-1$. Il suffit donc de démontrer que

$\left\|\mathcal{X}^{\beta_{j}} u \mathcal{X}^{\gamma_{j}} v\right\|_{H^{1}\left(\mathbb{H}^{n}\right)} \leq C\left(\|u\|_{L^{\infty}\left(\mathbb{H}^{n}\right)}\|v\|_{H^{k}\left(\mathbb{H}^{n}\right)}+\|v\|_{L^{\infty}\left(\mathbb{H}^{n}\right)}\|u\|_{H^{k}\left(\mathbb{H}^{n}\right)}\right)$.

Mais on a

$$
\mathcal{X}^{\beta_{j}} u \mathcal{X}^{\gamma_{j}} v=\sum_{q \geq 0}\left(S_{q} \mathcal{X}^{\beta_{j}} u\right)\left(\Delta_{q} \mathcal{X}^{\gamma_{j}} v\right)+\sum_{q \geq 0}\left(\Delta_{q} \mathcal{X}^{\beta_{j}} u\right)\left(S_{q+1} \mathcal{X}^{\gamma_{j}} v\right)
$$

et le Lemme 3.1 implique que

$$
\begin{aligned}
\left\|\left(S_{q} \mathcal{X}^{\beta_{j}} u\right)\left(\Delta_{q} \mathcal{X}^{\gamma_{j}} v\right)\right\|_{L^{2}} & \leq\left\|S_{q} \mathcal{X}^{\beta_{j}} u\right\|_{L^{\infty}}\left\|\Delta_{q} \mathcal{X}^{\gamma_{j}} v\right\|_{L^{2}} \\
& \leq C 2^{q \beta_{j}}\|u\|_{L^{\infty}} v_{q}\|v\|_{H^{k}} 2^{-q\left(k-\gamma_{j}\right)}
\end{aligned}
$$

où $\left\{v_{q}\right\}_{q \geq 0}$ est une suite de $\ell^{2}(\mathbb{N})$, de norme 1 . On peut alors conclure que

$$
\left\|\left(S_{q} \mathcal{X}^{\beta_{j}} u\right)\left(\Delta_{q} \mathcal{X}^{\gamma_{j}} v\right)\right\|_{L^{2}} \leq C v_{q} 2^{-q}\|u\|_{L^{\infty}}\|v\|_{H^{k}},
$$

ce qui démontre le résultat.

\subsubsection{Action des applications homogènes.}

La proposition suivante décrit l'action des applications homogènes dans les espaces de Besov, et est la traduction au groupe de Heisenberg [7, Théorème 1.3.2]. Avant d'énoncer le résultat, donnons la définition suivante.

Définition 3.1. Pour toute fonction $f \in C^{\infty}\left(\mathbb{R}^{*}\right)$, on appellera $f\left(\left(-\Delta_{\mathbb{H}^{n}}\right)^{1 / 2}\right)$ l'opérateur défini par

$$
\mathcal{F}\left(f\left(\left(-\Delta_{\mathbb{H}^{n}}\right)^{1 / 2}\right) u\right)(\lambda) F_{\alpha, \lambda}=f\left((4|\lambda|(2|\alpha|+n))^{1 / 2}\right) \mathcal{F}(u)(\lambda) F_{\alpha, \lambda},
$$

pour tout $u \in \mathcal{S}\left(\mathbb{H}^{n}\right)$. 
Proposition 3.1. Si $f \in C^{\infty}\left(\mathbb{R}^{*}\right)$ est homogène de degré $m$, alors pour tout $\rho<N / p$, pour tout $(p, r) \in[1, \infty]^{2}$ et pour tout $u \in \dot{B}_{p, r}^{\rho}$,

$$
\lim _{k \rightarrow \infty}\left\|\sum_{j \leq k} \Delta_{j} f\left(\left(-\Delta_{\mathbb{H} n}\right)^{1 / 2}\right) u\right\|_{\dot{B}_{p, r}^{\rho-m}} \leq C\|u\|_{\dot{B}_{p, r}^{\rho}} .
$$

DÉmonstration. Nous allons démontrer que pour toute fonction $u \in$ $\mathcal{S}\left(\mathbb{H}^{n}\right)$, on a

$$
\left\|f\left(\left(-\Delta_{\mathbb{H}^{n}}\right)^{1 / 2}\right) u\right\|_{\dot{B}_{p, r}^{\rho-m}} \leq C\|u\|_{\dot{B}_{p, r}^{\rho}} .
$$

La proposition suit alors par densité. Avec les notations rappellées au Paragraphe 2.2, on a

$$
\begin{aligned}
\mathcal{F}\left(\dot{\Delta}_{j} f((\right. & \left.\left.\left.-\Delta_{\mathbb{H}^{n}}\right)^{1 / 2}\right) u\right)(\lambda) F_{\alpha, \lambda} \\
& =R_{|\alpha|}^{*}\left(2^{-2 j} \lambda\right) f\left((4|\lambda|(2|\alpha|+n))^{1 / 2}\right) \mathcal{F}(u)(\lambda) F_{\alpha, \lambda},
\end{aligned}
$$

ce qui, par l'homogénéité de $f$, conduit à

$$
\begin{aligned}
\mathcal{F}\left(\dot{\Delta}_{j}\right. & \left.f\left(\left(-\Delta_{\mathbb{H}^{n}}\right)^{1 / 2}\right) u\right)(\lambda) F_{\alpha, \lambda} \\
& =2^{j m} R_{|\alpha|}^{*}\left(2^{-2 j} \lambda\right) f\left(\left(4\left|2^{-2 j} \lambda\right|(2|\alpha|+n)\right)^{1 / 2}\right) \mathcal{F}(u)(\lambda) F_{\alpha, \lambda} .
\end{aligned}
$$

Définissons alors $\theta^{*}(\lambda)=R^{*}(\lambda) f\left(2|\lambda|^{1 / 2}\right)$, et soit $h$ la fonction radiale, dans $\mathcal{S}\left(\mathbb{H}^{n}\right)$ par [4], telle que

$$
\mathcal{F}(h)(\lambda) F_{\alpha, \lambda}=\theta_{|\alpha|}^{*}(\lambda) F_{\alpha, \lambda},
$$

où comme précédemment, on a noté $\theta_{|\alpha|}^{*}(\lambda)=\theta^{*}((2|\alpha|+n) \lambda)$. La condition de support de $R^{*}$ nous permet d'écrire

$$
\begin{aligned}
\mathcal{F}\left(\dot{\Delta}_{j} f\left(\left(-\Delta_{\mathbb{H}^{n}}\right)^{1 / 2}\right) u\right)(\lambda) F_{\alpha, \lambda} & 2^{j m} \theta_{|\alpha|}^{*}\left(2^{-2 j} \lambda\right) \sum_{\left|j-j^{\prime}\right| \leq 1} R_{|\alpha|}^{*}\left(2^{-2 j^{\prime}} \lambda\right) \mathcal{F}(u)(\lambda) F_{\alpha, \lambda} .
\end{aligned}
$$

On en conclut que

$$
\dot{\Delta}_{j} f\left(\left(-\Delta_{\mathbb{H}^{n}}\right)^{1 / 2}\right) u=2^{j m+j N} h\left(\delta_{2^{j}} \cdot\right) * \sum_{\left|j-j^{\prime}\right| \leq 1} \dot{\Delta}_{j^{\prime}} u,
$$

et donc le lemme est démontré, par application de l'inégalité de Young comme dans [7]. 


\subsubsection{Composition par des fonctions $C^{\infty}$.}

Le Lemme 3.1 a enfin pour conséquence le résultat suivant, sur la composition par des fonctions de classe $C^{\infty}$.

Proposition 3.2. Soit $u \in L^{\infty} \cap B_{p, r}^{\rho}\left(\mathbb{H}^{n}\right)$ une fonction à valeurs réelles, avec $\rho>0$. Soit $F$ une fonction dans l'espace $C^{\infty}(\mathbb{R})$ telle que $F(0)=0$. Alors $F(u)$ est dans $L^{\infty} \cap B_{p, r}^{\rho}\left(\mathbb{H}^{n}\right)$, et

$$
\|F(u)\|_{B_{p, r}^{\rho}\left(\mathbb{H}^{n}\right)} \leq C\|u\|_{B_{p, r}^{\rho}\left(\mathbb{H}^{n}\right)},
$$

où $C$ ne dépend que de $F$ et de $\|u\|_{L^{\infty}\left(\mathbb{H}^{n}\right)}$.

DÉmonstration. La démonstration de cette proposition repose sur le lemme de caractérisation 3.2, et est identique au cas classique (voir [1], [13]). Rappellons brièvement la méthode: on écrit $F(u)$ comme la série

$$
F(u)=\sum_{j \geq-1} v_{j}, \quad \text { où } \quad v_{j}=F\left(\sum_{k \leq j} \Delta_{k} u\right)-F\left(\sum_{k \leq j-1} \Delta_{k} u\right),
$$

en se souvenant que

$$
F(u)=\lim _{j \rightarrow \infty} F\left(\sum_{k \leq j} \Delta_{k} u\right)
$$

Il suffit alors de démontrer que

$$
\left\|\left(-\Delta_{\mathbb{H}^{n}}\right)^{\gamma} v_{j}\right\|_{L^{p}\left(\mathbb{H}^{n}\right)} \leq C_{\gamma} c_{j} 2^{-j(\rho-2 \gamma)}, \quad \text { pour tout } \gamma \in \mathbb{N}
$$

où $C_{\gamma}$ ne dépend que de $\gamma$, et $\left\{c_{j}\right\}_{j \geq 0}$ est une suite de $\ell^{r}(\mathbb{N})$, ce qui par le Lemme 3.2 donnera le résultat.

L'estimation (3.15) s'obtient en écrivant la formule de Taylor avec reste intégral, à l'ordre 1 , qui fournit

$$
F\left(\sum_{k \leq j} \Delta_{k} u\right)-F\left(\sum_{k \leq j-1} \Delta_{k} u\right)=\Delta_{j} u \int_{0}^{1} F^{\prime}\left(\sum_{k \leq j-1} \Delta_{k} u+t \Delta_{j} u\right) d t
$$

La fin de la preuve consiste alors à estimer des dérivées successives du terme dans l'intégrale, par application de la formule de Faa-di-Bruno: nous renvoyons à [13] pour des détails. 
Remarque. On a le même type de résultat pour les espaces de Sobolev homogènes $\dot{H}^{s}$, pour tout $s>0$.

\subsubsection{Inclusions de Sobolev.}

Nous allons dans cette section présenter une démonstration des inclusions de Sobolev utilisant le découpage dyadique.

Théorème 3.1. Soit $p \in[1, \infty]$ et soit $\rho \in \mathbb{R}$ tel que $0<\rho<N / r$. Alors l'inclusion

$$
B_{r, r}^{\rho}\left(\mathbb{H}^{n}\right) \subset L^{p}\left(\mathbb{H}^{n}\right), \quad \text { avec } p=\frac{r N}{N-r \rho}
$$

est continue.

DÉmonstration. Soit $f \in \mathcal{S}\left(\mathbb{H}^{n}\right)$. On a

$$
\|f\|_{L^{p}\left(\mathbb{H}^{n}\right)}^{p}=p \int_{0}^{\infty} a^{p-1} \mu(\{|f|>a\}) d a,
$$

où $\mu$ est la mesure de Haar sur $\mathbb{H}^{n}$ (égale à la mesure de Lebesgue). Soit alors $A$ un réel strictement positif à fixer, et écrivons

$$
f=f_{1, A}+f_{2, A}, \quad \text { avec } f_{1, A}=\sum_{2^{j}<A} \Delta_{j} f \text { et } f_{2, A}=\sum_{2^{j} \geq A} \Delta_{j} f
$$

On a, en utilisant le Lemme 3.1,

$$
\begin{aligned}
\left\|f_{1, A}\right\|_{L^{\infty}\left(\mathbb{H}^{n}\right)} & \leq \sum_{2^{j}<A}\left\|\Delta_{j} f\right\|_{L^{\infty}\left(\mathbb{H}^{n}\right)} \\
& \leq \sum_{2^{j}<A} 2^{j \rho}\left\|\Delta_{j} f\right\|_{L^{r}\left(\mathbb{H}^{n}\right)} 2^{j(N / r-\rho)} \\
& \leq C A^{N / r-\rho}\|f\|_{B_{r, r}^{\rho}\left(\mathbb{H}^{n}\right)} .
\end{aligned}
$$

Choisissons à présent $A=A_{a}$ tel que

$$
C A_{a}^{N / r-\rho}\|f\|_{B_{r, r}^{\rho}\left(\mathbb{H}^{n}\right)}=\frac{a}{4} .
$$


Comme

$$
\mu(\{|f|>a\}) \leq \mu\left(\left\{\left|f_{1, A}\right|>\frac{a}{2}\right\}\right)+\mu\left(\left\{\left|f_{2, A}\right|>\frac{a}{2}\right\}\right),
$$

on en déduit, avec le choix $A=A_{a}$, que

$$
\mu(\{|f|>a\}) \leq \mu\left(\left\{\left|f_{2, A}\right|>\frac{a}{2}\right\}\right) .
$$

Mais on a, par l'inégalité de Bienaymé-Tchebytchev,

$$
\mu\left(\left\{\left|f_{2, A}\right|>\frac{a}{2}\right\}\right) \leq 2^{r} a^{-r}\left\|f_{2, A}\right\|_{L^{r}\left(\mathbb{H}^{n}\right)}^{r},
$$

et

$$
\begin{aligned}
\left\|f_{2, A_{a}}\right\|_{L^{r}\left(\mathbb{H}^{n}\right)}^{r} & =\int_{\mathbb{H} n}\left|\sum_{2^{j} \geq A_{a}} \Delta_{j} f\right|^{r} d z d s \\
& \leq \int_{\mathbb{H} n}\left(\sum_{2^{j} \geq A_{a}} 2^{j \varepsilon r}\left|\Delta_{j} f\right|^{r} d z d s\right)\left(\sum_{2^{j} \geq A_{a}} 2^{-j \varepsilon r^{\prime}}\right)^{r / r^{\prime}}
\end{aligned}
$$

où $1 / r+1 / r^{\prime}=1$, et donc

$$
\left\|f_{2, A_{a}}\right\|_{L^{r}\left(\mathbb{H}^{n}\right)}^{r} \leq C A_{a}^{-\varepsilon r} \sum_{2^{j} \geq A_{a}} 2^{j \varepsilon r}\left\|\Delta_{j} f\right\|_{L^{r}\left(\mathbb{H}^{n}\right)}^{r} .
$$

Par conséquent, on peut écrire, en utilisant le théorème de Fubini, que

$$
\begin{aligned}
\|f\|_{L^{p}\left(\mathbb{H}^{n}\right)}^{p} \leq & C \int_{0}^{\infty} a^{p-r-1} A_{a}^{-\varepsilon r} \sum_{2^{j} \geq A_{a}} 2^{j \varepsilon r}\left\|\Delta_{j} f\right\|_{L^{r}\left(\mathbb{H}^{n}\right)}^{r} d a \\
\leq & C \sum_{j \geq-1}\left(\int_{0}^{C 2^{j(N / r-\rho)}\|f\|_{B_{r, r}^{\rho}}} a^{p-r-1-\varepsilon r(r /(N-r \rho))} d a\right) \\
& \cdot\left(4 C\|f\|_{B_{r, r}^{\rho}}\right)^{\varepsilon r^{2}(N-r \rho)^{-1}} 2^{j \varepsilon r}\left\|\Delta_{j} f\right\|_{L^{r}\left(\mathbb{H}^{n}\right)}^{r} \\
\leq & C\|f\|_{B_{r, r}^{\rho}}^{p-r} \sum_{j \geq-1} 2^{j(N / r-\rho)(p-r)}\left\|\Delta_{j} f\right\|_{L^{r}\left(\mathbb{H}^{n}\right)}^{r} \\
\leq & C\|f\|_{B_{r, r}^{\rho}}^{p-r} \sum_{j \geq-1} 2^{j \rho r}\left\|\Delta_{j} f\right\|_{L^{r}\left(\mathbb{H}^{n}\right)}^{r} \cdot
\end{aligned}
$$


Le théorème est démontré.

REMARQUE. La même démonstration permet d'obtenir l'inégalité de Sobolev précisée suivante (voir [10])

$$
\|f\|_{L^{p}\left(\mathbb{H}^{n}\right)} \leq C\|f\|_{B_{\infty}^{-(N / \infty) \rho)}\left(\mathbb{H}^{n}\right)}^{1-r / p}\|f\|_{B_{r, r}^{p}\left(\mathbb{H}^{n}\right)}^{r / p} .
$$

Il suffit en effet de modifier le calcul (3.16): si l'on n'utilise pas le Lemme 3.1 , il vient

$$
\left\|f_{1, A}\right\|_{L^{\infty}} \leq C A^{N / r-\rho}\|f\|_{B_{\infty, \infty}^{-(N / r-\rho)}},
$$

et les calculs sont alors identiques, en choissant $A=A_{a}$ avec

$$
\frac{a}{4}=C A_{a}^{N / r-\rho}\|f\|_{B_{\infty, \infty}^{-(N / r-\rho)}} .
$$

\section{Paraproduit sur le groupe de Heisenberg.}

L'objet de cette section est d'adapter au groupe de Heisenberg l'algorithme de paraproduit introduit par J.-M. Bony dans [5].

Par rapport au cas classique, une difficulté apparaît, due au fait que l'on ne dispose pas d'écriture simple pour la transformée de Fourier du produit de deux fonctions. Notamment il n'est pas évident a priori, et contrairement au cas classique, que si deux fonctions ont une transformée de Fourier supportée dans des couronnes suffisamment éloignées l'une de l'autre, alors la transformée de Fourier de leur produit reste supportée dans une couronne. Néanmoins ce résultat est conservé pour le groupe de Heisenberg, comme le montre la proposition suivante.

Proposition 4.1. Soient $j$ et $j^{\prime}$ deux entiers, et soient $f$ et $g$ deux fonctions de $\mathcal{S}^{\prime}\left(\mathbb{H}^{n}\right)$ telles que $f * \widetilde{f}_{j}=0$ et $g * \widetilde{g}_{j^{\prime}}=0$ pour toutes les fonctions radiales $\widetilde{f}_{j}$ et $\widetilde{g}_{j^{\prime}}$ dans $\mathcal{S}\left(\mathbb{H}^{n}\right)$, telles que

$$
\begin{aligned}
\mathcal{F}\left(\tilde{f}_{j}\right)(\lambda) F_{\alpha, \lambda}=0, & \text { pour } \lambda \in(2|\alpha|+n)^{-1} 2^{2 j} \mathcal{C}_{0}, \\
\mathcal{F}\left(\widetilde{g}_{j^{\prime}}\right)(\lambda) F_{\alpha, \lambda}=0, & \text { pour } \lambda \in(2|\alpha|+n)^{-1} 2^{2 j^{\prime}} \mathcal{C}_{0} .
\end{aligned}
$$

Alors si $j^{\prime}-j>1$, il existe une couronne $\mathcal{C}_{0}^{\prime}$ telle que $f g * \widetilde{h}_{j^{\prime}}=0$ pour toutes les fonctions radiales $\widetilde{h}_{j^{\prime}}$ dans $\mathcal{S}\left(\mathbb{H}^{n}\right)$, telles que

$$
\mathcal{F}\left(\widetilde{h}_{j^{\prime}}\right)(\lambda) F_{\alpha, \lambda}=0, \quad \text { pour } \lambda \in(2|\alpha|+n)^{-1} 2^{2 j^{\prime}} \mathcal{C}_{0}^{\prime} .
$$


D'autre part, si $\left|j^{\prime}-j\right| \leq 1$, alors il existe une boule $\mathcal{B}_{0}^{\prime}$ telle que $f g *$ $\widetilde{h}_{j^{\prime}}^{\prime}=0$ pour toutes les fonctions radiales $\widetilde{h}_{j^{\prime}}^{\prime}$ dans $\mathcal{S}\left(\mathbb{H}^{n}\right)$, telles que

$$
\mathcal{F}\left(\widetilde{h}_{j^{\prime}}^{\prime}\right)(\lambda) F_{\alpha, \lambda}=0, \quad \text { pour } \lambda \in(2|\alpha|+n)^{-1} 2^{2 j^{\prime}} \mathcal{B}_{0}^{\prime} .
$$

Remarque. De la même manière que pour le Lemme 3.1 vu plus haut, dans le cas de fonctions dans $\mathcal{S}\left(\mathbb{H}^{n}\right)$, cette proposition s'écrit plus simplement de la façon suivante.

Proposition 4.2. Soient $j$ et $j^{\prime}$ deux entiers, et soient $f$ et $g$ deux fonctions de $\mathcal{S}\left(\mathbb{H}^{n}\right)$ telles que

$$
\begin{aligned}
& \mathcal{F}(f)(\lambda) F_{\alpha, \lambda}=\mathbf{1}_{(2|\alpha|+n)^{-1} 2^{2 j} \mathcal{C}_{0}}(\lambda) \mathcal{F}(f)(\lambda) F_{\alpha, \lambda}, \\
& \mathcal{F}(g)(\lambda) F_{\alpha, \lambda}=\mathbf{1}_{(2|\alpha|+n)^{-1} 2^{2 j^{\prime}} \mathcal{C}_{0}}(\lambda) \mathcal{F}(g)(\lambda) F_{\alpha, \lambda}
\end{aligned}
$$

avec $j^{\prime}-j>1$. Alors il existe une couronne $\mathcal{C}_{0}^{\prime}$ telle que

$$
\mathcal{F}(f g)(\lambda) F_{\alpha, \lambda}=\mathbf{1}_{(2|\alpha|+n)^{-1} 2^{2 j^{\prime}} \mathcal{C}_{0}^{\prime}}(\lambda) \mathcal{F}(f g)(\lambda) F_{\alpha, \lambda} .
$$

D'autre part, si $\left|j^{\prime}-j\right| \leq 1$, alors il existe une boule $\mathcal{B}_{0}^{\prime}$ telle que

$$
\mathcal{F}(f g)(\lambda) F_{\alpha, \lambda}=\mathbf{1}_{(2|\alpha|+n)^{-1} 2^{2 j^{\prime}} \mathcal{B}_{0}^{\prime}}(\lambda) \mathcal{F}(f g)(\lambda) F_{\alpha, \lambda} .
$$

Démonstration de la Proposition 4.1. Nous supposerons dans la suite que $f$ et $g$ sont deux fonctions de $\mathcal{S}\left(\mathbb{H}^{n}\right)$, la Proposition 4.1 s'obtenant par densité. On est donc ramené à démontrer la Proposition 4.2 .

Pour simplifier nous ne traiterons dans la suite que le cas $\lambda>0$. Par définition de $\mathcal{F}(f)(\lambda)$, on a

$$
\begin{aligned}
\mathcal{F}(f)(\lambda) F_{\alpha, \lambda}(\xi) & =\int_{\mathbb{H}^{n}} f(z, s) u_{z, s}^{\lambda} F_{\alpha, \lambda}(\xi) d z d s \\
& =\int_{\mathbb{H}^{n}} f(z, s) \frac{(\sqrt{2 \lambda}(\xi-\bar{z}))^{\alpha}}{\sqrt{\alpha !}} e^{i \lambda s+2 \lambda\left(\xi \cdot z-|z|^{2} / 2\right)} d z d s .
\end{aligned}
$$

En écrivant $\xi=\xi_{a}+i \xi_{b}$ et $z=z_{a}+i z_{b}$, il vient

$$
\left.\mathcal{F}(f)(\lambda) F_{\alpha, \lambda}(\xi)=\left(A_{\lambda, \xi}^{\alpha} f\right) \widehat{(}-2 \lambda \xi_{b},-2 \lambda \xi_{a},-\lambda\right),
$$


où l'on a écrit $\widehat{f}$ pour la transformée de Fourier usuelle de toute fonction $f$, et où

$$
A_{\lambda, \xi}^{\alpha} f(z, s)=\frac{(\sqrt{2 \lambda}(\xi-\bar{z}))^{\alpha}}{\sqrt{\alpha !}} e^{-\lambda\left(|\xi-\bar{z}|^{2}-|\xi|^{2}\right)} f(z, s) .
$$

REMARQUe. Par ce calcul, on a fait le lien entre la transformée de Fourier "Heisenberg" et la transformée de Fourier usuelle. C'est ce lien qui est la clef de la démonstration du résultat.

On peut à présent écrire

$$
\mathcal{F}(f g)(\lambda) F_{\alpha, \lambda}(\xi)=\left(A_{\lambda, \xi}^{\alpha} f g\right) \hat{(}\left(-2 \lambda \xi_{b},-2 \lambda \xi_{a},-\lambda\right) .
$$

Soit alors $\beta$ un multi-indice tel que $\beta \leq \alpha$, et $|\beta|=E(|\alpha| / 2)$, où $E$ est la partie entière. Définissons

$$
B_{\lambda, \xi}^{\beta} f(z, s)=\frac{(\sqrt{2 \lambda}(\xi-\bar{z}))^{\beta}}{\sqrt{\beta !}} f(z, s) .
$$

Alors on a

$$
\begin{aligned}
& \left(A_{\lambda, \xi}^{\alpha} f g\right) \hat{(}\left(-2 \lambda \xi_{b},-2 \lambda \xi_{a},-\lambda\right) \\
& \quad=\left(\begin{array}{c}
\alpha \\
\beta
\end{array}\right)^{-1 / 2}\left(B_{\lambda, \xi}^{\beta} f \widehat{)} *\left(A_{\lambda, \xi}^{\alpha-\beta} g\right) \widehat{\tau}\left(-2 \lambda \xi_{b},-2 \lambda \xi_{a},-\lambda\right) .\right.
\end{aligned}
$$

Il reste donc à étudier les supports de ces deux fonctions en convolution. On sait que

$$
\begin{aligned}
\left(A_{\lambda, \xi}^{\alpha-\beta} g\right) \widetilde{(} & \left.-2 \lambda \xi_{b},-2 \lambda \xi_{a},-\lambda\right) \\
& =\mathcal{F}(g)(\lambda) F_{\alpha-\beta, \lambda}(\xi) \\
& =\mathbf{1}_{(2|\alpha-\beta|+n)^{-1} 2^{2 j^{\prime}} \mathcal{C}_{0}}(\lambda)\left(A_{\lambda, \xi}^{\alpha-\beta} g\right) \hat{\Upsilon}\left(-2 \lambda \xi_{b},-2 \lambda \xi_{a},-\lambda\right)
\end{aligned}
$$

donc le support en $\lambda$ de la fonction $\left(A_{\lambda, \xi}^{\alpha-\beta} g(z, s) \widetilde{\Upsilon}\left(-2 \lambda \xi_{b},-2 \lambda \xi_{a},-\lambda\right)\right.$ est inclus dans la couronne $(2|\alpha-\beta|+n)^{-1} 2^{2 j^{\prime}} \mathcal{C}_{0}$.

Lemme 4.1. La fonction $\left(B_{\lambda, \xi}^{\beta} f\right)^{\Upsilon}\left(-2 \lambda \xi_{b},-2 \lambda \xi_{a},-\lambda\right)$ vérifie

$$
\begin{aligned}
&\left(B_{\lambda, \xi}^{\beta} f\right)\left(-2 \lambda \xi_{b},-2 \lambda \xi_{a},-\lambda\right) \\
& \quad=\mathbf{1}_{(2|\beta|+n)^{-1} 2^{2 j} \mathcal{B}_{0}^{\prime \prime}}(\lambda)\left(B_{\lambda, \xi}^{\beta} f\right) \widetilde{(}\left(-2 \lambda \xi_{b},-2 \lambda \xi_{a},-\lambda\right)
\end{aligned}
$$


où $\mathcal{B}_{0}^{\prime \prime}=\{\tau \in \mathbb{R}:|\tau| \leq 4\}$.

Supposons un instant ce lemme démontré. Alors la Proposition 4.1 suit immédiatement, puisque le fait que $j^{\prime}-j>1$ implique que la couronne $\mathcal{C}_{0}$ et la boule $\mathcal{B}_{0}^{\prime \prime}$ sont disjointes. De même, on a le résultat cherché dans le cas où $\left|j^{\prime}-j\right| \leq 1$.

DÉmonstration DU LEMme 4.1. Écrivons

$$
\begin{aligned}
&\left(B_{\lambda, \xi}^{\beta} f\right)^{\widehat{ }}(\left.-2 \lambda \xi_{b},-2 \lambda \xi_{a},-\lambda\right) \\
&= \int f(z, s) e^{i \lambda s+2 i \lambda\left(\xi_{b} z_{a}+\xi_{a} z_{b}\right)} \frac{(\sqrt{2 \lambda}(\xi-\bar{z}))^{\beta}}{\sqrt{\beta !}} d z d s \\
&= \int f(z, s) e^{-\lambda\left(|\xi-\bar{z}|^{2}-|\xi|^{2}\right)} \frac{(\sqrt{2 \lambda}(\xi-\bar{z}))^{\beta}}{\sqrt{\beta !}} \\
& \cdot e^{\lambda\left(|\xi-\bar{z}|^{2}-|\xi|^{2}\right)} e^{i J_{\lambda}(s, z, \xi)} d z d s,
\end{aligned}
$$

avec $J_{\lambda}(s, z, \xi)=\lambda s+2 \lambda\left(\xi_{b} z_{a}+\xi_{a} z_{b}\right)$. Mais il existe une suite $\left\{c_{k}\right\}_{k \in \mathbb{N}^{n}}$ telle que

$$
e^{\lambda|\xi-\bar{z}|^{2}}=\sum_{k \in \mathbb{N}^{n}} c_{k}\left(\prod_{i=1}^{n}\left(\bar{\xi}_{i}-z_{i}\right)^{k_{i}}\right) \frac{\lambda^{|k|}(\xi-\bar{z})^{k}}{(2 k) !} .
$$

La fonction $\left(B_{\lambda, \xi}^{\beta} f\right)^{\Upsilon}\left(-2 \lambda \xi_{b},-2 \lambda \xi_{a},-\lambda\right)$ est donc égale à

$$
\begin{gathered}
\int e^{i J_{\lambda}(s, z, \xi)} e^{-\lambda\left(|\xi-\bar{z}|^{2}-|\xi|^{2}\right)} \frac{(\sqrt{2 \lambda}(\xi-\bar{z}))^{\beta}}{\sqrt{\beta !}} \\
\cdot f(z, s) \sum_{k \in \mathbb{N}^{n}} c_{k}\left(\prod_{i=1}^{n}\left(\bar{\xi}_{i}-z_{i}\right)^{k_{i}}\right) \frac{\lambda^{|k|}(\xi-\bar{z})^{k}}{(2 k) !} d z d s .
\end{gathered}
$$

En écrivant

$$
\frac{(\sqrt{2 \lambda}(\xi-\bar{z}))^{\beta} \lambda^{|k|}(\xi-\bar{z})^{k}}{\sqrt{\beta !}(2 k) !}=\frac{(\sqrt{2 \lambda}(\xi-\bar{z}))^{\beta+k}}{\sqrt{(\beta+k) !}} \lambda^{|k| / 2} d_{k, \beta},
$$

où les $d_{k, \beta}$ sont des constantes, il vient pour $\left(B_{\lambda, \xi}^{\beta} f\right)\left(-2 \lambda \xi_{b},-2 \lambda \xi_{a}\right.$, $-\lambda)$

$$
\begin{aligned}
\sum_{k \in \mathbb{N}^{n}} \lambda^{|k| / 2} d_{k, \beta} c_{k} \int & e^{i J_{\lambda}(s, z, \xi)} f(z, s)\left(\prod_{i=1}^{n}\left(\bar{\xi}_{i}-z_{i}\right)^{k_{i}}\right) \\
& \cdot e^{-\lambda\left(|\xi-\bar{z}|^{2}-|\xi|^{2}\right)} \frac{(\sqrt{2 \lambda}(\xi-\bar{z}))^{\beta+k}}{\sqrt{(\beta+k) !}} d z d s,
\end{aligned}
$$


d'où finalement

$$
\left(B_{\lambda, \xi}^{\beta} f \widetilde{)^{-}}=\sum_{k \in \mathbb{N}^{n}} \lambda^{|k| / 2} d_{k, \beta} c_{k}\left(A_{\lambda, \xi}^{\beta+k}\left(\prod_{i=1}^{n}\left(\bar{\xi}_{i}-z_{i}\right)^{k_{i}}\right) \hat{f}\right) .\right.
$$

Étudions séparément chacun des termes de cette série. Il est facile de voir que pour tout $\gamma$ tel que $\gamma_{i} \neq 0$,

$$
\begin{aligned}
\partial_{\xi_{i}}(\mathcal{F}(f)(\lambda)) & F_{\gamma, \lambda}(\xi) \\
& =c_{\gamma} \sqrt{\lambda} \mathcal{F}(f)(\lambda) F_{\gamma-1_{i}, \lambda}(\xi)+2 \lambda\left(\mathcal{F}\left(z_{i} f\right)(\lambda)\right) F_{\gamma, \lambda}(\xi)
\end{aligned}
$$

où l'on a noté $1_{i}$ pour le vecteur de $\mathbb{R}^{n}$ dont toutes les composantes sont nulles sauf la composante $i$, égale à 1 . Dans le cas où $\gamma_{i}=0$, on a simplement

$$
\partial_{\xi_{i}}(\mathcal{F}(f)(\lambda)) F_{\gamma, \lambda}(\xi)=2 \lambda\left(\mathcal{F}\left(z_{i} f\right)(\lambda)\right) F_{\gamma, \lambda}(\xi)
$$

Donc le support en $\lambda$ de $\mathcal{F}\left(\left(\bar{\xi}_{i}-z_{i}\right) f\right)(\lambda) F_{\gamma, \lambda}(\xi)$ est inclus dans la réunion suivante

$$
2^{2 j}(2|\gamma|+n)^{-1} \mathcal{C}_{0} \cup 2^{2 j}(2(|\gamma|-1)+n)^{-1} \mathcal{C}_{0}
$$

Une récurrence immédiate implique que le support en $\lambda$ de

$$
\mathcal{F}\left(\prod_{i=1}^{n}\left(\bar{\xi}_{i}-z_{i}\right)^{k_{i}} f\right)(\lambda) F_{\gamma, \lambda}(\xi)
$$

est inclus dans

$$
2^{2 j}(2|\gamma|+n)^{-1} \mathcal{C}_{0} \cup \cdots \cup 2^{2 j}\left(2\left(|\gamma|-k_{1}-\cdots-k_{n}\right)+n\right)^{-1} \mathcal{C}_{0}
$$

Mais comme $\gamma=\beta+k$, on obtient finalement que le support en $\lambda$ de

$$
\left(A_{\lambda, \xi}^{\beta+k}\left(\prod_{i=1}^{n}\left(\bar{\xi}_{i}-z_{i}\right)^{k_{i}}\right) \hat{f}\right) \hat{\left(-2 \lambda \xi_{b},-2 \lambda \xi_{a},-\lambda\right)}
$$

est inclus dans

$$
2^{2 j}(2|\beta|+n)^{-1} \mathcal{C}_{0} \cup \cdots \cup 2^{2 j}\left(2\left(|\beta|+k_{1}+\cdots+k_{n}\right)+n\right)^{-1} \mathcal{C}_{0},
$$


c'est-à-dire dans la boule, indépendante de $k$,

$$
(2|\beta|+n)^{-1} 2^{2 j} \mathcal{B}_{0}^{\prime \prime}, \quad \text { où } \quad \mathcal{B}_{0}^{\prime \prime}=\{\tau \in \mathbb{R}:|\tau| \leq 4\} .
$$

Mais alors chacun des termes de la série, qui converge vers

$$
\left(B_{\lambda, \xi}^{\beta} f \widetilde{)}\left(-2 \lambda \xi_{b},-2 \lambda \xi_{a},-\lambda\right)\right.
$$

est supporté dans une boule fixe, ce qui implique que

$$
\left(B_{\lambda, \xi}^{\beta} f\right)^{\widehat{(}}\left(-2 \lambda \xi_{b},-2 \lambda \xi_{a},-\lambda\right)
$$

est supporté dans cette même boule.

Le lemme est donc démontré, et avec lui, la Proposition 4.1.

\subsection{L'algorithme de paraproduit.}

\subsubsection{Définitions.}

Définition 4.1. On appelle paraproduit de $f$ par $g$, et l'on note $T_{f} g$, l'opérateur bilinéaire suivant

$$
T_{f} g \stackrel{\text { def }}{=} \sum_{j^{\prime} \leq j-2} \Delta_{j^{\prime}} f \Delta_{j} g=\sum_{j} S_{j-1} f \Delta_{j} g,
$$

où l'on a défini

$$
S_{j} f=\sum_{j^{\prime} \leq j-1} \Delta_{j^{\prime}} f
$$

On appelle reste du produit $f g$, et l'on note $R(f, g)$, l'opérateur bilinéaire symétrique suivant

$$
R(f, g) \stackrel{\text { def }}{=} \sum_{\left|j-j^{\prime}\right| \leq 1} \Delta_{j^{\prime}} f \Delta_{j} g .
$$

Remarque. La Proposition 4.1 implique en particulier que pour tout $j \geq 0$ et pour tout $\mu \in\{-1,0,1\}$, on a

$$
\begin{aligned}
\mathcal{F}\left(S_{j-1} f \Delta_{j} g\right)(\lambda) F_{\alpha, \lambda} & =\mathbf{1}_{(2|\alpha|+n)^{-1} 2^{2 j} \mathcal{C}_{0}^{\prime}}(\lambda) \mathcal{F}\left(S_{j-1} f \Delta_{j} g\right)(\lambda) F_{\alpha, \lambda} \\
\mathcal{F}\left(\Delta_{j-\mu} f \Delta_{j} g\right)(\lambda) F_{\alpha, \lambda} & =\mathbf{1}_{(2|\alpha|+n)^{-1} 2^{2 j} \mathcal{B}_{0}^{\prime}}(\lambda) \mathcal{F}\left(S_{j-\mu} f \Delta_{j} g\right)(\lambda) F_{\alpha, \lambda} .
\end{aligned}
$$




\subsubsection{Lois de produit.}

Les démonstrations des résultats ci-dessous s'obtiennent exactement comme dans le cas classique, par application du Lemme 3.1. Nous renvoyons à [7] pour les détails.

Théorème 4.1. Soient $\rho$ et $\rho^{\prime}$ deux réels, et $p$ et $r$ deux éléments de $[1,+\infty]$. Alors si a est un élément de $L^{\infty}\left(\mathbb{H}^{n}\right)$, l'opérateur $T_{a}$ est continu de $B_{p, r}^{\rho}$ dans $B_{p, r}^{\rho}$, et si $a \in C^{\rho^{\prime}}\left(\mathbb{H}^{n}\right)$ avec $\rho^{\prime}<0$, alors $T_{a}$ est continu de $B_{p, r}^{\rho}$ dans $B_{p, r}^{\rho+\rho^{\prime}}$, et l'on a

$$
\left\|T_{a} b\right\|_{B_{p, r}^{\rho}\left(\mathbb{H}^{n}\right)} \leq C\|a\|_{L^{\infty}\left(\mathbb{H}^{n}\right)}\|b\|_{B_{p, r}^{\rho}\left(\mathbb{H}^{n}\right)},
$$

et

$$
\left\|T_{a} b\right\|_{B_{p, r}^{\rho+\rho^{\prime}}\left(\mathbb{H}^{n}\right)} \leq C\|a\|_{C \rho^{\prime}\left(\mathbb{H}^{n}\right)}\|b\|_{B_{p, r}^{\rho}\left(\mathbb{H}^{n}\right)}, \quad \rho^{\prime}<0 .
$$

D'autre part, pour tous réels $\rho_{1}$ et $\rho_{2}$ tels que $\rho_{1}+\rho_{2}>0$, et pour tous les $p_{1}, p_{2}, p, r_{1}, r_{2}$ dans $[1,+\infty]$ tels que $1 / p \leq 1 / p_{1}+1 / p_{2}$ et $1 / r \stackrel{\text { def }}{=}$ $1 / r_{1}+1 / r_{2} \leq 1$, l'opérateur $R$ est bilinéaire continu de

$$
B_{p_{1}, r_{1}}^{\rho_{1}} \times B_{p_{2}, r_{2}}^{\rho_{2}} \quad \text { dans } \quad B_{p, r}^{\rho_{12}}
$$

où

$$
\rho_{12}=\rho_{1}+\rho_{2}-N\left(\frac{1}{p_{1}}+\frac{1}{p_{2}}-\frac{1}{p}\right) .
$$

Enfin si $\rho_{1}+\rho_{2} \geq 0$ et $1 / p \leq 1 / p_{1}+1 / p_{2}$ et $1 / r_{1}+1 / r_{2}=1$, alors $R$ est bilinéaire continu de

$$
B_{p_{1}, r_{1}}^{\rho_{1}} \times B_{p_{2}, r_{2}}^{\rho_{2}} \quad \text { dans } \quad B_{p, \infty}^{\rho_{12}}
$$

Corollaire 4.1. Soient $\rho>0$ et $(p, r) \in[1,+\infty]^{2}$ trois réels. Si u et $v$ sont deux éléments de $L^{\infty} \cap B_{p, r}^{\rho}\left(\mathbb{H}^{n}\right)$, alors $u v \in B_{p, r}^{\rho}\left(\mathbb{H}^{n}\right)$, et

$$
\|u v\|_{B_{p, r\left(\mathbb{H}^{n}\right)}^{\rho}} \leq C\left(\|u\|_{L^{\infty}}\|v\|_{B_{p, r}^{\rho}}+\|v\|_{L^{\infty}}\|u\|_{B_{p, r}^{\rho}}\right) .
$$

Si $\rho_{1}+\rho_{2}>0$ et si $p_{1}$ est tel que $\rho_{1}<N / p_{1}$, alors pour tout couple $\left(p_{2}, r_{2}\right) \in[1,+\infty]^{2}$, on a pour tous $u$ et $v$ dans $B_{p_{1}, \infty}^{\rho_{1}} \cap B_{p_{2}, r_{2}}^{\rho_{2}}\left(\mathbb{H}^{n}\right)$,

$$
\|u v\|_{B_{p_{2}, r_{2}}^{\rho}\left(\mathbb{H}^{n}\right)} \leq C\left(\|u\|_{B_{p_{1}, \infty}^{\rho_{1}}}\|v\|_{B_{p_{2}, r_{2}}^{\rho_{2}}}+\|v\|_{B_{p_{1}, \infty}^{\rho_{1}}}\|u\|_{B_{p_{2}, r_{2}}^{\rho_{2}}}\right),
$$


où $\rho=\rho_{1}+\rho_{2}-N / p_{1}$. D'autre part, si $\rho_{1}+\rho_{2} \geq 0, \rho_{1}<N / p_{1}$ et $1 / r_{1}+1 / r_{2}=1$, alors pour $u$ et $v$ dans $B_{p_{1}, r_{1}}^{\rho_{1}} \cap B_{p_{2}, r_{2}}^{\rho_{2}}\left(\mathbb{H}^{n}\right)$, on a

$$
\|u v\|_{B_{p, \infty}^{\rho}\left(\mathbb{H}^{n}\right)} \leq C\left(\|u\|_{B_{p_{1}, r_{1}}^{\rho_{1}}}\|v\|_{B_{p_{2}, r_{2}}^{\rho_{2}}}+\|v\|_{B_{p_{1}, r_{1}}^{\rho_{1}}}\|u\|_{B_{p_{2}, r_{2}}^{\rho_{2}}}\right) .
$$

Enfin si $\rho_{1}+\rho_{2}>0, \rho_{j}<N / p_{j}$ et $p \geq \max \left\{p_{1}, p_{2}\right\}$, alors pour tout $\left(r_{1}, r_{2}\right)$,

$$
\|u v\|_{B_{p, r}^{\rho_{1}\left(\mathbb{H}^{n}\right)}} \leq C\|u\|_{B_{p_{1}, r_{1}}^{\rho_{1}}}\|v\|_{B_{p_{2}, r_{2}}^{\rho_{2}}}
$$

avec

$$
\rho_{12}=\rho_{1}+\rho_{2}-N\left(\frac{1}{p_{1}}+\frac{1}{p_{2}}-\frac{1}{p}\right)
$$

et $r=\max \left\{r_{1}, r_{2}\right\}$, et si $\rho_{1}+\rho_{2} \geq 0$, avec $\rho_{j}<N / p_{j}$ et $1 / r_{1}+1 / r_{2}=1$, alors pour tout $p \geq \max \left\{p_{1}, p_{2}\right\}$,

$$
\|u v\|_{B_{p, \infty}^{\rho_{12}\left(\mathbb{H}^{n}\right)}} \leq C\|u\|_{B_{p_{1}, r_{1}}^{\rho_{1}}}\|v\|_{B_{p_{2}, r_{2}}^{\rho_{2}}} .
$$

REMARQUE. Les résultats correspondant au cas des espaces de Besov homogènes s'énoncent de manière identique.

Un second corollaire à ce théorème est démontré dans [8], la démonstration ici est identique.

Proposition 4.3. Soient $\rho$ et $r$ deux réels tels que $\rho \geq 2$ et $1 / 2<r<$ 1. Il existe alors une constante $C$ telle que pour toutes fonctions $u, v$, et $w$ avec

$$
u \in B_{1, \infty}^{\rho} \cap C^{r}\left(\mathbb{H}^{n}\right) \quad \text { et } \quad(v, w) \in\left(B_{1, \infty}^{\rho-1} \cap C^{r-1}\right)^{2}\left(\mathbb{H}^{n}\right),
$$

on $a$

$$
\|u v w\|_{B_{1, \infty}^{\rho-2+r}\left(\mathbb{H}^{n}\right)} \leq C\|u\|_{B_{1, \infty}^{\rho} \cap C^{r}}\|v\|_{B_{1, \infty}^{\rho-1} \cap C^{r-1}}\|w\|_{B_{1, \infty}^{\rho-1} \cap C^{r-1}} .
$$

Enfin les opérateurs de paraproduit permettent de préciser la Proposition 3.2 , sur la composition par une fonction $C^{\infty}$, de la manière suivante.

Proposition 4.3. Soit u une fonction à valeurs réelles telle que $u \in$ $B_{p, r}^{\rho}\left(\mathbb{H}^{n}\right)$, avec $\rho>N / p$. Soit enfin $F \in C^{\infty}(\mathbb{R})$. Alors

$$
F(u)=T_{F^{\prime}(u)} u+R, \quad \text { ò̀ } \quad R \in B_{p, r}^{2 \rho-N / p}\left(\mathbb{H}^{n}\right) .
$$


DÉmonstration. Nous n'allons pas donner ici les détails des calculs conduisant à ce résultat, et renvoyons au livre de Y. Meyer ([13]) pour la démonstration de cette proposition. Rappellons simplement que la démonstration consiste à écrire la série téléscopique (3.14) déjà employée pour démontrer la Proposition 3.2, et d'utiliser alors la formule de Taylor avec reste intégral à l'ordre deux.

\section{Applications.}

\subsection{L'inégalité de Gagliardo-Nirenberg.}

On définit les espaces $W^{\sigma, r}\left(\mathbb{H}^{n}\right)$ comme la complétion de $\mathcal{S}\left(\mathbb{H}^{n}\right)$ pour la norme

$$
\|u\|_{W^{\sigma, r}\left(\mathbb{H}^{n}\right)}=\left\|\left(-\Delta_{\mathbb{H}^{n}}\right)^{\sigma / 2} u\right\|_{L^{r}\left(\mathbb{H}^{n}\right)} \cdot
$$

L'objet de cette section est de démontrer, par application des résultats présentés précédemment, le théorème suivant.

Théorème 5.1. Soit $f$ une fonction de $L^{q} \cap W^{\sigma, r}\left(\mathbb{H}^{n}\right)$, avec $q$ et $r$ strictement supérieurs à 1 et $\sigma \geq 0$. Alors $f \in W^{\rho, p}\left(\mathbb{H}^{n}\right)$, et

$$
\left\|\left(-\Delta_{\mathbb{H}^{n}}\right)^{\rho / 2} f\right\|_{L^{p}\left(\mathbb{H}^{n}\right)} \leq C\|f\|_{L^{q}\left(\mathbb{H}^{n}\right)}^{\theta}\left\|\left(-\Delta_{\mathbb{H}^{n}}\right)^{\sigma / 2} f\right\|_{L^{r}\left(\mathbb{H}^{n}\right)}^{1-\theta},
$$

ò̀ $1 / p=\theta / q+(1-\theta) / r, \rho=(1-\theta) \sigma$, et $\theta \in] 0,1[$.

DÉmonstration. Commençons par rappeller la définition et les principales propriétés de la fonction maximale (voir [18, Chapitre XIII, p. 638], pour des détails).

Rappellons que la distance homogène sur le groupe de Heisenberg est définie par

$$
\||(z, s)|\| \stackrel{\text { def }}{=}\left(|z|^{4}+|s|^{2}\right)^{1 / 4} .
$$

Les "boules" associées à cette distance sont notées

$$
B(z, s, R)=\left\{\left(z^{\prime}, s^{\prime}\right) \in \mathbb{H}^{n}:\left\|\left(z^{\prime}, s^{\prime}\right)(z, s)^{-1}\right\| \leq R\right\}
$$

et leur mesure est notée $m(B(z, s, R))$. 
Définition 5.1. Soit $f \in L_{\mathrm{loc}}^{1}\left(\mathbb{H}^{n}\right)$. La fonction maximale de $f$ est définie par

$$
M f(z, s) \stackrel{\text { def }}{=} \sup _{R>0} \frac{1}{m(B(z, s, R))} \int_{B(z, s, R)}\left|f\left(z^{\prime}, s^{\prime}\right)\right| d z^{\prime} d s^{\prime} .
$$

Proposition 5.1. Si $f \in L^{p}\left(\mathbb{H}^{n}\right)$, avec $1<p \leq \infty$, alors $M f \in$ $L^{p}\left(\mathbb{H}^{n}\right)$, et

$$
\|M f\|_{L^{p}\left(\mathbb{H}^{n}\right)} \leq A_{p}\|f\|_{L^{p}\left(\mathbb{H}^{n}\right)}
$$

où $A_{p}$ est une constante dépendant de $p$ et de $n$.

D'autre part, soit $\varphi \in L^{1}\left(\mathbb{H}^{n}\right)$, et supposons que le plus petit majorant radial de $\varphi$, noté $\psi$ et défini par

$$
\psi(z, s)=\sup _{\left\|\left(z^{\prime}, s^{\prime}\right)\right\| \geq\|\|(z, s) \|} \varphi\left(z^{\prime}, s^{\prime}\right)
$$

est dans $L^{1}\left(\mathbb{H}^{n}\right)$. Alors pour tout $f \in L^{p}\left(\mathbb{H}^{n}\right)$, avec $1 \leq p \leq \infty$, on a

$$
|f * \varphi(z, s)| \leq\|\psi\|_{L^{1}\left(\mathbb{H}^{n}\right)} M f(z, s) \text {. }
$$

Démontrons à présent l'inégalité proposée pour une fonction $f \in$ $\mathcal{S}\left(\mathbb{H}^{n}\right)$. On peut écrire

$$
\begin{aligned}
& \left(-\Delta_{\mathbb{H}^{n}}\right)^{\rho / 2} f \\
& \quad=\sum_{j \leq A}\left(-\Delta_{\mathbb{H}^{n}}\right)^{\rho / 2} \dot{\Delta}_{j} f+\sum_{j>A}\left(-\Delta_{\mathbb{H}^{n}}\right)^{(\rho-\sigma) / 2} \dot{\Delta}_{j}\left(\left(-\Delta_{\mathbb{H}^{n}}\right)^{(\sigma / 2)} f\right),
\end{aligned}
$$

où $A$ est une constante à fixer. Nous avons vu en (3.12) que

$$
\begin{aligned}
& \mathcal{F}\left(a\left(\left(-\Delta_{\mathbb{H} n}\right)^{1 / 2}\right) \dot{\Delta}_{j} f\right)(\lambda) F_{\alpha, \lambda} \\
& \quad=2^{j m} R_{|\alpha|}^{*}\left(2^{-2 j} \lambda\right) a\left(\left(4\left|2^{-2 j \lambda}\right|(2|\alpha|+n)\right)^{1 / 2}\right) \mathcal{F}(f)(\lambda) F_{\alpha, \lambda},
\end{aligned}
$$

dès que $a \in C^{\infty}\left(\mathbb{R}^{*}\right)$ est homogène de degré $m$. Alors comme en (3.13), on a

$$
a\left(-\Delta_{\mathbb{H}^{n}}\right) \dot{\Delta}_{j} f=2^{j m+j N} h\left(\delta_{2^{j}} \cdot\right) * \sum_{\left|j-j^{\prime}\right| \leq 1} \dot{\Delta}_{j^{\prime}} f,
$$


où $h$ est la fonction de $\mathcal{S}\left(\mathbb{H}^{n}\right)$ telle que

$$
\mathcal{F}(h)(\lambda) F_{\alpha, \lambda}=R_{|\alpha|}^{*}(\lambda) a\left(\left(4\left|2^{-2 j} \lambda\right|\right)^{1 / 2}\right) F_{\alpha, \lambda} .
$$

Mais alors comme dans [2], il existe une fonction $\widetilde{h}$ radiale, intégrable et décroissante en la distance à l'origine, qui majore $h$, ce qui par application de (5.2), donne

$$
\left|a\left(-\Delta_{\mathbb{H} n}\right) \dot{\Delta}_{j} f(z, s)\right| \leq C 2^{j m} M f(z, s) .
$$

En appliquant cette inégalité à $a(D)=\left(-\Delta_{\mathbb{H}^{n}}\right)^{\rho / 2}$ puis à $a\left(-\Delta_{\mathbb{H}^{n}}\right)=$ $\left(-\Delta_{\mathbb{H}^{n}}\right)^{(\rho-\sigma) / 2}$, il vient

$$
\begin{array}{rl}
\mid\left(-\Delta_{\mathbb{H} n}\right)^{\rho / 2} & f(z, s) \mid \\
& \leq C\left(\sum_{j \leq A} 2^{\rho j} M f(z, s)+\sum_{j>A} 2^{(\rho-\sigma) j} M\left(\left(-\Delta_{\mathbb{H}^{n}}\right)^{\sigma / 2} f\right)(z, s)\right) \\
& \leq C 2^{\rho A} M f(z, s)+C 2^{(\rho-\sigma) A} M\left(\left(-\Delta_{\mathbb{H}^{n}}\right)^{\sigma / 2} f\right)(z, s),
\end{array}
$$

puisque $\sigma>\rho$. En optimisant sur $A$, il vient

$$
\left|\left(-\Delta_{\mathbb{H} n}\right)^{\rho / 2} f(z, s)\right| \leq C(M f(z, s))^{1-\rho / \sigma}\left(M\left(\left(-\Delta_{\mathbb{H} n}\right)^{\sigma / 2} f\right)(z, s)\right)^{\rho / \sigma} .
$$

Il suffit alors d'appliquer l'inégalité de Hölder, qui donne

$$
\left\|\left(-\Delta_{\mathbb{H}^{n}}\right)^{\rho / 2} f\right\|_{L^{p}\left(\mathbb{H}^{n}\right)} \leq C\|M f\|_{L^{q}\left(\mathbb{H}^{n}\right)}^{\theta}\left\|M\left(\left(-\Delta_{\mathbb{H}^{n}}\right)^{\sigma / 2} f\right)\right\|_{L^{r}}^{1-\theta},
$$

avec $\theta=1-\rho / \sigma$.

L’inégalité maximale (5.1) termine la démonstration.

Remarque. Dans le cas où $p=q=r=1$, l'inégalité correspondante

$$
\left\|\left(-\Delta_{\mathbb{H}^{n}}\right)^{\rho / 2} f\right\|_{L^{1}\left(\mathbb{H}^{n}\right)} \leq C\|f\|_{L^{1}\left(\mathbb{H}^{n}\right)}^{\theta}\left\|\left(-\Delta_{\mathbb{H}^{n}}\right)^{\sigma / 2} f\right\|_{L^{1}\left(\mathbb{H}^{n}\right)}^{1-\theta},
$$

où $\rho=(1-\theta) \sigma$, et $\theta \in] 0,1[$, se démontre simplement par le calcul suivant

$$
\begin{aligned}
\left\|\left(-\Delta_{\mathbb{H}^{n}}\right)^{\rho / 2} f\right\|_{L^{1}\left(\mathbb{H}^{n}\right)} \leq & \left\|\sum_{j \leq A}\left(-\Delta_{\mathbb{H}^{n}}\right)^{\rho / 2} \dot{\Delta}_{j} f\right\|_{L^{1}\left(\mathbb{H}^{n}\right)} \\
& +\left\|\sum_{j>A}\left(-\Delta_{\mathbb{H}^{n}}\right)^{(\rho-\sigma) / 2} \dot{\Delta}_{j}\left(\left(-\Delta_{\mathbb{H}^{n}}\right)^{\sigma / 2} f\right)\right\|_{L^{1}\left(\mathbb{H}^{n}\right)} \\
\leq & C 2^{(1-\theta) \sigma A}\|f\|_{L^{1}\left(\mathbb{H}^{n}\right)} \\
& +C 2^{-\theta \sigma A}\left\|\left(-\Delta_{\mathbb{H}^{n}}\right)^{\sigma / 2} f\right\|_{L^{1}\left(\mathbb{H}^{n}\right)}
\end{aligned}
$$


ce qui, en optimisant sur $A$, conduit au résultat.

\section{2. Équations semi-linéaires sous-elliptiques.}

Dans [21], C.-J. Xu et C. Zuily démontrent un résultat de régularité des solutions faibles d'équations quasi-linéaires sous-elliptiques. Nous nous proposons ici, dans le cas où l'opérateur sous-elliptique est $-\Delta_{\mathbb{H} n}$ et dans un cadre semi-linéaire, d'en présenter une démonstration plus élémentaire, reposant sur le paraproduit et les espaces de Besov. Cette démonstration dans le cas classique est due à J.-Y. Chemin et C.-J. Xu, voir [8].

Considérons donc l'équation semi-linéaire sous-elliptique suivante, où $N_{0} \in \mathbb{N}$, et $b_{i j, k \ell}^{k^{\prime}}$ est une fonction indéfiniment différentiable

$$
-\Delta_{\mathbb{H}^{n}} u^{k^{\prime}}+\sum_{i, j=1}^{2 n} \sum_{k, \ell=1}^{N_{0}} b_{i j, k \ell}^{k^{\prime}}(u) X_{i} u^{k} X_{j} u^{\ell}=0,
$$

pour $k^{\prime} \in\left\{1, \ldots, N_{0}\right\}$. On peut alors démontrer le théorème suivant.

Théorème 5.2. Si $\rho$ est un réel tel que $\rho>1 / 2$, et si u est solution faible de (5.3) telle que $u \in H^{1}\left(\mathbb{H}^{n}\right) \cap C^{\rho}\left(\mathbb{H}^{n}\right)$, alors $u \in C^{\infty}\left(\mathbb{H}^{n}\right)$.

DÉmonstration. Les résultats obtenus jusqu'ici permettent de reprendre à l'identique la démonstration de [8]; nous la reproduisons ici pour la commodité du lecteur.

On peut supposer que $\rho \leq 1$. Commençons par remarquer que si $u \in H^{1}\left(\mathbb{H}^{n}\right) \cap C^{\rho}\left(\mathbb{H}^{n}\right)$, alors

$$
b_{i j, k \ell}^{k^{\prime}}(u) X_{i} u^{k} X_{j} u^{\ell} \in L^{1}\left(\mathbb{H}^{n}\right),
$$

et donc par l'injection continue de $L^{1}\left(\mathbb{H}^{n}\right)$ dans $B_{1, \infty}^{0}\left(\mathbb{H}^{n}\right)$, on en déduit que

$$
\Delta_{\mathbb{H}^{n}} u^{k^{\prime}} \in B_{1, \infty}^{0}\left(\mathbb{H}^{n}\right) .
$$

Mais l'opérateur $\left(-\Delta_{\mathbb{H}^{n}}\right)$ est un isomorphisme de $B_{p, r}^{\sigma}\left(\mathbb{H}^{n}\right)$ dans $B_{p, r}^{\sigma-2}\left(\mathbb{H}^{n}\right)$ pour tout $\sigma \in \mathbb{R}$, et pour tous $(p, r) \in[1, \infty]$ (voir [4]), par conséquent on obtient que $u \in B_{1, \infty}^{2}\left(\mathbb{H}^{n}\right)$.

On raisonne alors par récurrence, en montrant que pour tout $k \in \mathbb{N}$,

$$
u \in B_{1, \infty}^{2+k \rho} \cap C^{\rho}\left(\mathbb{H}^{n}\right) \quad \text { implique } \quad u \in B_{1, \infty}^{2+(k+1) \rho}\left(\mathbb{H}^{n}\right),
$$


ce qui démontrera le théorème.

Pour démontrer (5.4), il suffit d'utiliser la Proposition 3.2 ci-dessus, qui implique que

$$
b_{i j, k \ell}^{k^{\prime}}(u) \in B_{1, \infty}^{2+k \rho} \cap C^{\rho}\left(\mathbb{H}^{n}\right),
$$

et donc par le Corollaire 4.2, on a

$$
b_{i j, k \ell}^{k^{\prime}}(u) X_{i} u^{k} X_{j} u^{\ell} \in B_{1, \infty}^{(k+1) \rho}\left(\mathbb{H}^{n}\right) .
$$

On en conclut alors que

$$
u \in B_{1, \infty}^{2+(k+1) \rho}\left(\mathbb{H}^{n}\right)
$$

ce qui achève la démonstration.

\section{3. Équations d'ondes semi-linéaires.}

Considérons l'équation d'ondes semi-linéaire suivante

$$
\left\{\begin{array}{l}
\partial_{t t} u-\Delta_{\mathbb{H}^{n}} u=|X u|^{2} F(u), \quad \text { dans } \mathbb{R} \times \mathbb{H}^{n} \\
\left(u_{\mid t=0}, \partial_{t} u_{\mid t=0}\right)=\left(u_{0}, u_{1}\right)
\end{array}\right.
$$

où $F \in C^{\infty}(\mathbb{R})$. On a noté $X u=\left(X_{1} u, \ldots, X_{2 n} u\right)$, et l'on notera dorénavant $D u=\left(\partial_{t} u, X u\right)$. Démontrons le théorème suivant.

Théorème 5.3. Soit $s>N / 2+3 / 4$, et $\left(u_{0}, u_{1}\right) \in \dot{H}^{s} \times \dot{H}^{s-1}\left(\mathbb{H}^{n}\right)$. Alors il existe un temps $T>0$ tel que (5.5) possède une unique solution $u$, avec

$$
u \in L^{\infty}\left([0, T], \dot{H}^{s}\left(\mathbb{H}^{n}\right)\right),
$$

et

$$
D u \in L^{\infty}\left([0, T], \dot{H}^{s-1}\left(\mathbb{H}^{n}\right)\right) \cap L^{4}\left([0, T], L^{\infty}\left(\mathbb{H}^{n}\right)\right)
$$

Remarque. Ce théorème est l'analogue sur le groupe de Heisenberg du [17, Théorème 1] du cas classique. Notons toutefois que la restriction sur l'indice $s$ est plus forte dans notre cadre (quand $d=3$, la restriction dans [17] est $s>2$ ). Comme nous le verrons dans la démonstration, cela est dû au fait que le domaine de validité des estimations de Strichartz 
généralisées, sur le groupe de Heisenberg, est moins étendu sur $\mathbb{H}^{n}$ que sur $R^{n}$ (voir [4]).

DÉmonstration DU ThÉORÈME. Nous allons commencer par rappeler [4, Théorème 4.1], donnant les estimations de Strichartz généralisées, sur le groupe de Heisenberg, vérifiées par la solution $u$ de

$$
\left\{\begin{array}{l}
\partial_{t t} u-\Delta_{\mathbb{H}^{n}} u=f, \quad \text { dans } \mathbb{R} \times \mathbb{H}^{n}, \\
\left(u_{\mid t=0}, \partial_{t} u_{\mid t=0}\right)=\left(u_{0}, u_{1}\right) .
\end{array}\right.
$$

Notons que dans [4], le théorème est démontré pour $\left(u_{0}, u_{1}\right) \in \dot{H}^{1} \times$ $L^{2}\left(\mathbb{H}^{n}\right)$, mais on obtient de manière identique le cas $\left(u_{0}, u_{1}\right) \in \dot{H}^{s} \times$ $\dot{H}^{s-1}\left(\mathbb{H}^{n}\right)$.

Proposition 5.2. Soient trois réels $s, \rho_{1}$ et $\rho_{2}$, et soient $p_{i}, r_{i}$, pour $i \in$ $\{1,2\}$, tels que

$$
\begin{aligned}
& \frac{2}{p_{i}} \leq \frac{1}{2}-\frac{1}{r_{i}} \quad \text { et } \quad 2 \leq r_{i} \leq \infty, \\
& \rho_{1}+N\left(\frac{1}{2}-\frac{1}{r_{1}}\right)-\frac{1}{p_{1}}=s \quad \text { et } \quad \rho_{2}+N\left(\frac{1}{2}-\frac{1}{r_{2}}\right)-\frac{1}{p_{2}}=1-s .
\end{aligned}
$$

Supposons que $\left(u_{0}, u_{1}\right) \in \dot{H}^{s} \times \dot{H}^{s-1}\left(\mathbb{H}^{n}\right)$. Alors pour tout temps $T$, on $a$

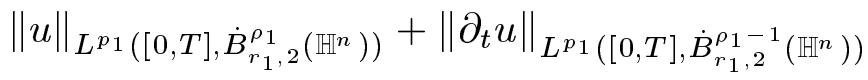

$$
\begin{aligned}
& \leq C\left\|\left(u_{0}, u_{1}\right)\right\|_{\dot{H}^{s} \times \dot{H}^{s-1}\left(\mathbb{H}^{n}\right)}+C\|f\|_{L^{\bar{p}_{2}}\left([0, T], \dot{B}_{\bar{r}_{2}, 2}^{-\rho_{2}}\left(\mathbb{H}^{n}\right)\right)},
\end{aligned}
$$

où pour tout $r$, on a noté $\bar{r}$ pour son conjugué, défini par $1 / r+1 / \bar{r}=1$.

Pour alléger les notations, on notera $L_{T}^{p}\left(\dot{B}_{p, r}^{\rho}\left(\mathbb{H}^{n}\right)\right)$ l'espace $L^{p}\left([0, T], \dot{B}_{p, r}^{\rho}\left(\mathbb{H}^{n}\right)\right)$. D'autre part, on supposera dans la suite que $s \leq N / 2+1$ puisque dans le cas $s>N / 2+1$, la résolution du système est simplement due à la théorie classique des systèmes symétriques hyperboliques. Par la proposition ci-dessus, on a donc

$$
\begin{aligned}
& \|u\|_{L_{T}^{\infty}\left(\dot{H}^{s}\left(\mathbb{H}^{n}\right)\right)}+\left\|\partial_{t} u\right\|_{L_{T}^{\infty}\left(\dot{H}^{s-1}\left(\mathbb{H}^{n}\right)\right)} \\
& \quad \leq C\left\|\left(u_{0}, u_{1}\right)\right\|_{\dot{H}^{s} \times \dot{H}^{s-1}\left(\mathbb{H}^{n}\right)}+C\left\||X u|^{2} F(u)\right\|_{L_{T}^{1}\left(\dot{H}^{s-1}\left(\mathbb{H}^{n}\right)\right)},
\end{aligned}
$$


et, pour tout $\varepsilon>0$, puisque $s>N / 2+3 / 4$, on a

$$
\begin{aligned}
& \|u\|_{L_{T}^{p_{1}}\left(\dot{B}_{\infty, 2}^{\varepsilon+1}\left(\mathbb{H}^{n}\right)\right)}+\left\|\partial_{t} u\right\|_{L_{T}^{p_{1}}\left(\dot{B}_{\infty, 2}^{\varepsilon}\left(\mathbb{H}^{n}\right)\right)} \\
& \leq C\left\|\left(u_{0}, u_{1}\right)\right\|_{\dot{H}^{s} \times \dot{H}^{s-1}\left(\mathbb{H}^{n}\right)}+C\left\||X u|^{2} F(u)\right\|_{L_{T}^{1}\left(\dot{H}^{s-1}\left(\mathbb{H}^{n}\right)\right)},
\end{aligned}
$$

avec

$$
\frac{1}{p_{1}}=1+\varepsilon+\frac{N}{2}-s,
$$

et l'on peut choisir $p_{1}=4$.

Remarque. C'est ici que la restriction sur $s$ intervient, et elle est due au fait que sur le groupe de Heisenberg, on a nécessairement $p_{1} \geq 4$, alors que dans le cas classique, la limitation sur $p_{1}$ est $p_{1} \geq 2$ (en dimension $d \geq 3$ ). Cette limitation dans le cas classique permet donc de résoudre notre problème pour $s>(n+1) / 2$ dès que la dimension d'espace est $n \geq 3$, et pour $s>1+3 / 4$ en dimension 2 . Ici en toute dimension, on demande que

$$
s>\frac{N}{2}+\frac{3}{4}
$$

Le Lemme 3.3 indique que les $X_{j}$ opèrent sur les espaces de Besov, par conséquent l'estimation (5.6) s'écrit aussi

$$
\begin{aligned}
& \|D u\|_{L_{T}^{4}\left(\dot{B}_{\infty, 2}^{\varepsilon}\left(\mathbb{H}^{n}\right)\right)} \\
& \quad \leq C\left\|\left(u_{0}, u_{1}\right)\right\|_{\dot{H}^{s} \times \dot{H}^{s-1}\left(\mathbb{H}^{n}\right)}+C\left\||X u|^{2} F(u)\right\|_{L_{T}^{1}\left(\dot{H}^{s-1}\left(\mathbb{H}^{n}\right)\right)} .
\end{aligned}
$$

En outre, comme $\dot{B}_{\infty, 2}^{\varepsilon} \subset L^{\infty}$, on a finalement

$$
\begin{aligned}
& \|D u\|_{L_{T}^{4}\left(L^{\infty}\left(\mathbb{H}^{n}\right)\right)} \\
& \quad \leq C\left\|\left(u_{0}, u_{1}\right)\right\|_{\dot{H}^{s} \times \dot{H}^{s-1}\left(\mathbb{H}^{n}\right)}+C\left\||X u|^{2} F(u)\right\|_{L_{T}^{1}\left(\dot{H}^{s-1}\left(\mathbb{H}^{n}\right)\right)} .
\end{aligned}
$$

Il reste à estimer $|X u|^{2} F(u)$ dans $\dot{H}^{s-1}\left(\mathbb{H}^{n}\right)$. Pour cela, on va faire appel aux estimations douces obtenues en Section 3.3.2 et à la Proposition 3.2 sur la composition par des fonctions $C^{\infty}$, ainsi qu'à l'algorithme de paraproduit introduit dans la Définition 4.1. Commençons par définir la fonction $G \in C^{\infty}(\mathbb{R})$ par

$$
G(u)=F(u)-F(0) .
$$


Alors, par les estimations douces de la Section 3.3.2, on a

$$
\begin{aligned}
& \left\||X u|^{2} F(u)\right\|_{\dot{H}^{s-1}\left(\mathbb{H}^{n}\right)} \\
& \quad \leq C\|X u\|_{L^{\infty}\left(\mathbb{H}^{n}\right)}\|X u\|_{\dot{H}^{s-1}\left(\mathbb{H}^{n}\right)}+\left\||X u|^{2} G(u)\right\|_{\dot{H}^{s-1}\left(\mathbb{H}^{n}\right)} .
\end{aligned}
$$

Démontrons finalement le lemme suivant:

Lemme 5.1. Si $X u \in L^{\infty}$ et $u \in \dot{H}^{s}\left(\mathbb{H}^{n}\right)$, avec $s>N / 2$, alors

$$
\left\||X u|^{2} G(u)\right\|_{\dot{H}^{s-1}\left(\mathbb{H}^{n}\right)} \leq C\|X u\|_{L^{\infty}\left(\mathbb{H}^{n}\right)}\|u\|_{\dot{H}^{s}\left(\mathbb{H}^{n}\right)}^{2} .
$$

DÉmonstration du Lemme. Commençons par remarquer que la Proposition 3.2 sur la composition par des fonctions $C^{\infty}$ implique, puisque $G(0)=0$, que

$$
\|G(u)\|_{\dot{H}^{s}} \leq C\|u\|_{\dot{H}^{s}} .
$$

En outre, les estimations douces de la Section 3.2.2 fournissent

$$
\begin{aligned}
\left\||X u|^{2} G(u)\right\|_{\dot{H}^{s-1}\left(\mathbb{H}^{n}\right)} \leq & C\|X u\|_{\dot{H}^{s-1}\left(\mathbb{H}^{n}\right)}\||X u| G(u)\|_{L^{\infty}\left(\mathbb{H}^{n}\right)} \\
& +\|X u\|_{L^{\infty}\left(\mathbb{H}^{n}\right)}\||X u| G(u)\|_{\dot{H}^{s-1}\left(\mathbb{H}^{n}\right)}
\end{aligned}
$$

Le premier terme de cette estimation se majore en utilisant que

$$
\||X u| G(u)\|_{L^{\infty}\left(\mathbb{H}^{n}\right)} \leq C\|X u\|_{L^{\infty}\left(\mathbb{H}^{n}\right)}\|G(u)\|_{\dot{H}^{s}\left(\mathbb{H}^{n}\right)},
$$

puisque $s>N / 2$, ce qui donne

$$
\|X u\|_{\dot{H}^{s-1}\left(\mathbb{H}^{n}\right)}\||X u| G(u)\|_{L^{\infty}\left(\mathbb{H}^{n}\right)} \leq C\|u\|_{\dot{H}^{s}\left(\mathbb{H}^{n}\right)}^{2}\|X u\|_{L^{\infty}\left(\mathbb{H}^{n}\right)} .
$$

Quant au second terme, on écrit l'algorithme de paraproduit

$$
|X u| G(u)=T_{G(u)}|X u|+T_{|X u|} G(u)+R(G(u),|X u|) .
$$

Le Théorème 4.1 implique que

$$
\begin{aligned}
\left\|T_{G(u)}|X u|\right\|_{\dot{H}^{s-1}\left(\mathbb{H}^{n}\right)} & \leq C\|G(u)\|_{L^{\infty}\left(\mathbb{H}^{n}\right)}\|X u\|_{\dot{H}^{s-1}\left(\mathbb{H}^{n}\right)} \\
& \leq C\|u\|_{\dot{H}^{s}\left(\mathbb{H}^{n}\right)}^{2} .
\end{aligned}
$$


De même, on peut écrire que

$$
\begin{aligned}
\left\|T_{|X u|} G(u)\right\|_{\dot{H}^{s-1}\left(\mathbb{H}^{n}\right)} & \leq C\|X u\|_{\dot{C}^{-1}\left(\mathbb{H}^{n}\right)}\|G(u)\|_{\dot{H}^{s}\left(\mathbb{H}^{n}\right)} \\
& \leq C\|u\|_{\dot{H}^{s}\left(\mathbb{H}^{n}\right)}\|G(u)\|_{\dot{H}^{s}\left(\mathbb{H}^{n}\right)},
\end{aligned}
$$

puisque $s>N / 2$, ce qui donne l'estimation voulue. Enfin pour le terme de reste, on écrit que

$$
\begin{aligned}
\|R(G(u),|X u|)\|_{\dot{H}^{s-1}\left(\mathbb{H}^{n}\right)} & \leq C\|X u\|_{\dot{H}^{s-1}\left(\mathbb{H}^{n}\right)}\|G(u)\|_{\dot{B}_{\infty, \infty}^{0}\left(\mathbb{H}^{n}\right)} \\
& \leq C\|u\|_{\dot{H}^{s}\left(\mathbb{H}^{n}\right)}\|G(u)\|_{L^{\infty}\left(\mathbb{H}^{n}\right)} .
\end{aligned}
$$

Le lemme est donc démontré.

On peut à présent achever la démonstration de la proposition. En désignant $\|\cdot\|_{s, T}$ pour la norme

$$
\|u\|_{s, T}=\|u\|_{L_{T}^{\infty}\left(\dot{H}^{s}\left(\mathbb{H}^{n}\right)\right)}+\left\|\partial_{t} u\right\|_{L_{T}^{\infty}\left(\dot{H}^{s-1}\left(\mathbb{H}^{n}\right)\right)}+\|D u\|_{L_{T}^{4}\left(L^{\infty}\left(\mathbb{H}^{n}\right)\right)},
$$

on a finalement montré que

$$
\left.\|u\|_{s, T} \leq C\left\|\left(u_{0}, u_{1}\right)\right\|_{\dot{H}^{s} \times \dot{H}^{s-1}\left(\mathbb{H}^{n}\right)}+C\|u\|_{s, T}^{2} T^{3 / 4}+C\|u\|_{s, T}^{3} T^{3 / 4}\right) .
$$

Il est classique que ce type d'estimation conduit à l'existence de solutions en temps petit (dépendant de $\left(u_{0}, u_{1}\right)$ ). L'unicité est une conséquence du fait que

$$
D u \in L^{1}\left([0, T], L^{\infty}\left(\mathbb{H}^{n}\right)\right) .
$$

Remerciements. Nous remercions J.-Y. Chemin de nous avoir communiqué la démonstration du Théorème 3.1, ainsi que P. Gérard pour de nombreuses discussions et pour la preuve du Théorème 5.1 dans le cas classique.

\section{References.}

[1] Alinhac, S., Gérard, P., Opérateurs pseudo-différentiels et théorème de Nash-Moser, Savoirs Actuels. Interéditions, 1991. 
[2] Bahouri, H., Chemin, J.-Y., Équations de transport relatives à des champs de vecteurs non lipschitziens et mécanique des fluides. Arch. Rational Mech. Anal. 127 (1994), 159-182.

[3] Bahouri, H., Gérard, P., Xu, C.-J., Estimations de Strichartz géneralisées sur le groupe de Heisenberg. Séminaire EDP de l'École Polytechnique 10, 1997-1998.

[4] Bahouri, H., Gérard, P., Xu, C.-J., Espaces de Besov et estimations de Strichartz géneralisées sur le groupe de Heisenberg. Prépublication du département de Mathématiques de Paris-Sud, 1999. À paraître, Journal d'Analyse Mathématique.

[5] Bony, J.-M., Calcul symbolique et propagation des singularités pour les équations aux dérivées partielles non linéaires. Ann. Sci. École Norm. Sup. 14 (1981), 209-246.

[6] Chemin, J.-Y., Fluides parfaits incompressibles. Astérisque 230, 1995.

[7] Chemin, J.-Y., About the Navier-Stokes System. Prépublication du Laboratoire d'Analyse Numérique de Paris 6, 1997.

[8] Chemin, J.-Y., Xu, C.-J., Remarque sur la régularité de solutions faibles d'équations elliptiques semi-linéaires. C. R. Acad. Sci. Paris $\mathbf{3 2 5}$ (1997), 257-260.

[9] Geller, D., Fourier analysis on the Heisenberg group. Proc. Nat. Acad. Sci. USA 74 (1997), 1328-1331.

[10] Gérard, P., Meyer, Y., Oru, F., Inégalités de Sobolev précisées. Séminaire EDP de l'École Polytechnique 4, 1996-1997.

[11] Hulanicki, A., A functional calculus for Rockland operators on nilpotent Lie groups. Studia Math. 78 (1984), 253-266.

[12] Lemarié, P. G., Base d'ondelettes sur les groupes de Lie stratifiés. Bull. Soc. Math. France 117 (1989), 211-232.

[13] Meyer, Y., Ondelettes et opérateurs. Tome 3, Hermann, 1991.

[14] Müller, D., Stein, E. M., $L^{p}$-estimates for the wave equation on the Heisenberg group. Bericht. Math. Sem. Kiel (1997), 1-29.

[15] Nachman, A. I., The wave equation on the Heisenberg group. Comm. Partial Diff. Equations 7 (1982), 675-714.

[16] Nessibi, M., Sifi, M., Trimeche, K., Inversion of the Radon transform on the Laguerre hypergroup by using generalized wavelets. J. Math. Anal. Appl. 208 (1997), 337-363.

[17] Ponce, G., Sideris, T. C., Local regularity of nonlinear wave equations in three space dimensions. Comm. Partial Diff. Equations 18 (1993), 169-177.

[18] Stein, E. M., Harmonic Analysis. Princeton, 1992. 
[19] Taylor, M. E., Noncommutative Harmonic Analysis. Mathematical Surveys and Monographs, 22, AMS, 1986.

[20] Xu, C.-J., Nonlinear Microlocal Analysis. Workshop on General Theory of Partial Differential Equations. Trieste, 1995.

[21] Xu, C.-J., Zuily, C., Higher interior regularity for quasilinear subelliptic systems. Calc. Var. Partial Differ. Equations 5 (1997), 323-343.

Recibido: 15 de abril de 1.999

Hajer Bahouri Département de Mathématiques Faculté des Sciences de Tunis 1060 Tunis, TUNISIE Hajer.Bahouri@fst.rnu.tn

et

Isabelle Gallagher Département de Mathématiques Université de Paris-Sud 91405 Orsay Cedex, FRANCE Isabelle.Gallagher@math.u-psud.fr 